Revue des patrimoines

\title{
Une ferme modèle en territoire helvétique : le domaine de Beau-Cèdre à Jouxtens-Mézery (Vaud)
}

Isabelle Roland

\section{(2) OpenEdition}

Journals

Édition électronique

URL : http://journals.openedition.org/insitu/10324

DOI : $10.4000 /$ insitu. 10324

ISSN : 1630-7305

Éditeur

Ministère de la culture

Référence électronique

Isabelle Roland, « Une ferme modèle en territoire helvétique : le domaine de Beau-Cèdre à JouxtensMézery (Vaud) », In Situ [En ligne], 21 | 2013, mis en ligne le 21 novembre 2013, consulté le 19 avril 2019. URL : http://journals.openedition.org/insitu/10324; DOI : 10.4000/insitu.10324

Ce document a été généré automatiquement le 19 avril 2019

\section{(c) $($ i) $(9)$}

In Situ Revues des patrimoines est mis à disposition selon les termes de la licence Creative Commons Attribution - Pas d'Utilisation Commerciale - Pas de Modification 4.0 International. 


\title{
Une ferme modèle en territoire helvétique : le domaine de Beau-Cèdre à Jouxtens-Mézery (Vaud)
}

\author{
Isabelle Roland
}

\section{Portrait du propriétaire-constructeur : Gustave Auberjonois (1837-1917)}

1 Gustave Auberjonois, issu d'une famille de notables attestée dès le Moyen Age à Yverdon, est le fils de Victor Auberjonois, préfet de Lausanne, et de Clémence Perdonnet. Il passa son enfance entre Lausanne et les domaines ruraux que possédait son père, dont celui de Montagny-sur-Yverdon, ancienne seigneurie acquise par ses ancêtres. Après des études d'agronomie effectuées vraisemblablement à Zurich, il partit se perfectionner à l'étranger, séjournant dans des fermes en Allemagne, en Angleterre et en France. Le 8 août 1863, il acheta le domaine de la Grosse Grange à Jouxtens-Mézery, qu'il transforma selon ses propres plans à partir de 1872, tout en le rebaptisant du nom de Beau-Cèdre. En 1864, il épousa Pauline d'Albis, originaire de l'Aveyron, qui lui donna cinq enfants, dont le célèbre peintre René Auberjonois (1872-1957), trois filles et un second fils, Maurice (1875-1902), agronome et entomologiste, qui décéda malheureusement prématurément par noyade.

Outre ses activités d'agronome et de propriétaire terrien, Gustave Auberjonois s'intéressa à la politique et fut député du Grand Conseil vaudois. Il présida également la Société d'agriculture de la Suisse romande et la Société vaudoise d'horticulture. En 1910, on le nomma président d'honneur de l'Exposition nationale d'agriculture qui se tint à Lausanne. Sensible aux arts, il fut l'un des fondateurs du théâtre de cette ville, en 1871, et encouragea vivement la carrière artistique de son fils René. Dans le Dictionnaire historique et biographique de la Suisse, paru en 1921, soit quatre ans après son décès, il fut précisé que 
«ses constructions rurales et sa laiterie modèle de Beau-Cèdre ont acquis un renom même à l'étranger ».

Gustave Auberjonois partageait sont temps entre sa résidence lausannoise et Beau-Cèdre, où il vivait en véritable gentleman-farmer : « Toute une communauté villageoise vivait de cette ferme. Je revois encore le grand patron en haut-de-forme et canne à pommeau d'argent, les grandes dames à falbalas descendant des landaus, les cochers, les palefreniers, les gens de maison, et jusqu'au moutonnier, sous sa houppelande [...]. Quand j'y repense aujourd'hui, ce temps-là me fait songer à une sorte de haute société aristocratique attardée en plein $\mathrm{XX}^{\mathrm{e}}$ siècle ${ }^{1}$ ».

\section{Construction de la ferme de Beau-Cèdre, dispositions générales}

4 Lorsque Gustave Auberjonois acquit le domaine de la Grosse Grange, en 1863, celui-ci comprenait une maison de maître, datant du milieu du XVIII ${ }^{e}$ siècle, et deux dépendances abritant le logement du fermier, des granges et des écuries (fig. $\mathbf{n}^{\circ} \mathbf{1}$ ). La maison de maître étant fondée sur un sol instable, Gustave Auberjonois la fit reconstruire plus à l'ouest en 1872, en l'agrémentant d'un jardin à l'anglaise ${ }^{2}\left(\right.$ fig. $\left.^{\circ}{ }^{\circ} 2\right)$. Les bâtiments agricoles furent élevés progressivement de 1877 à 1888 , une seconde porcherie, non prévue dans le plan initial, venant compléter l'ensemble en 1891.

Figure 1

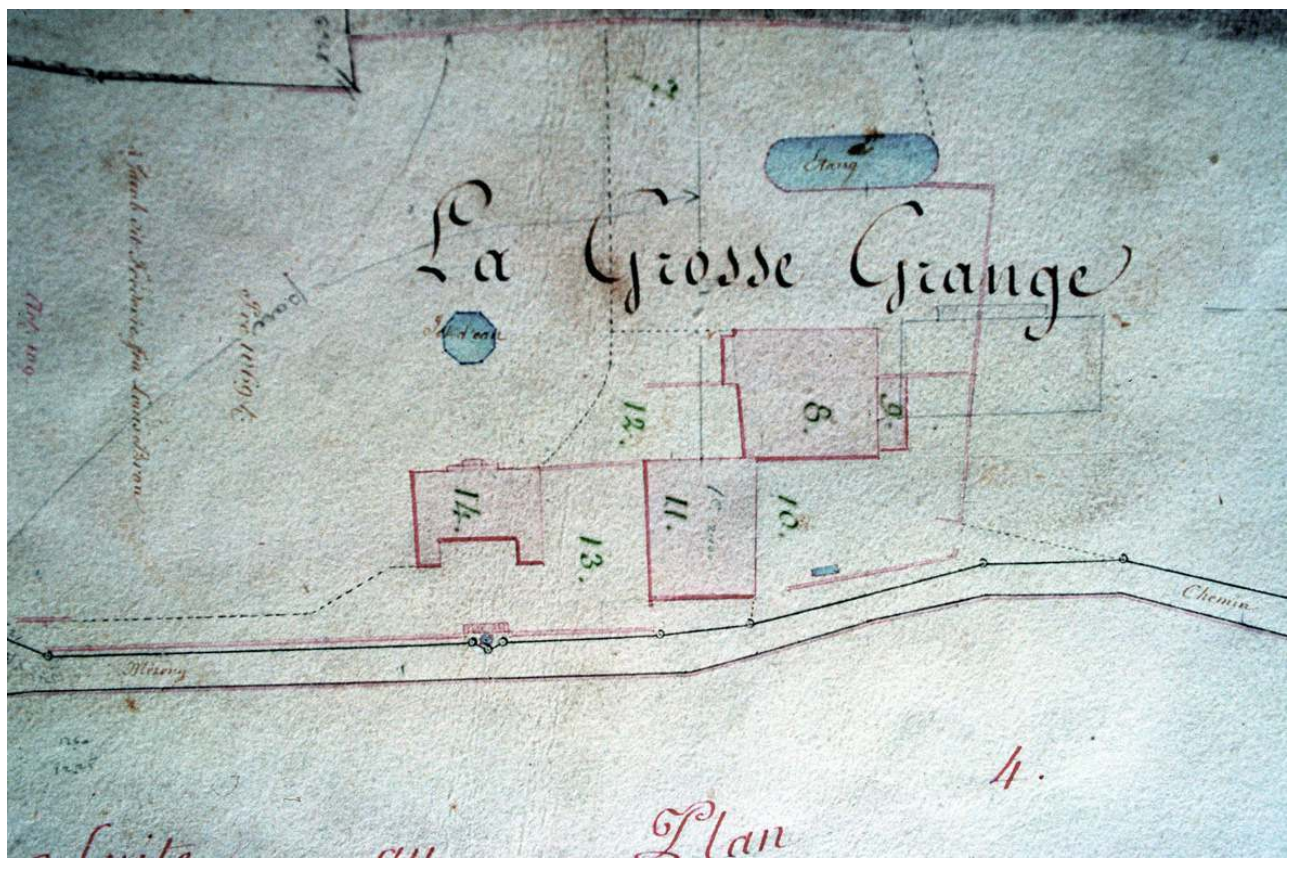

Plan cadastral de Jouxtens-Mézery, 1848, folio 14, nº 14 (ACV, GB 131 e). 
Figure 2

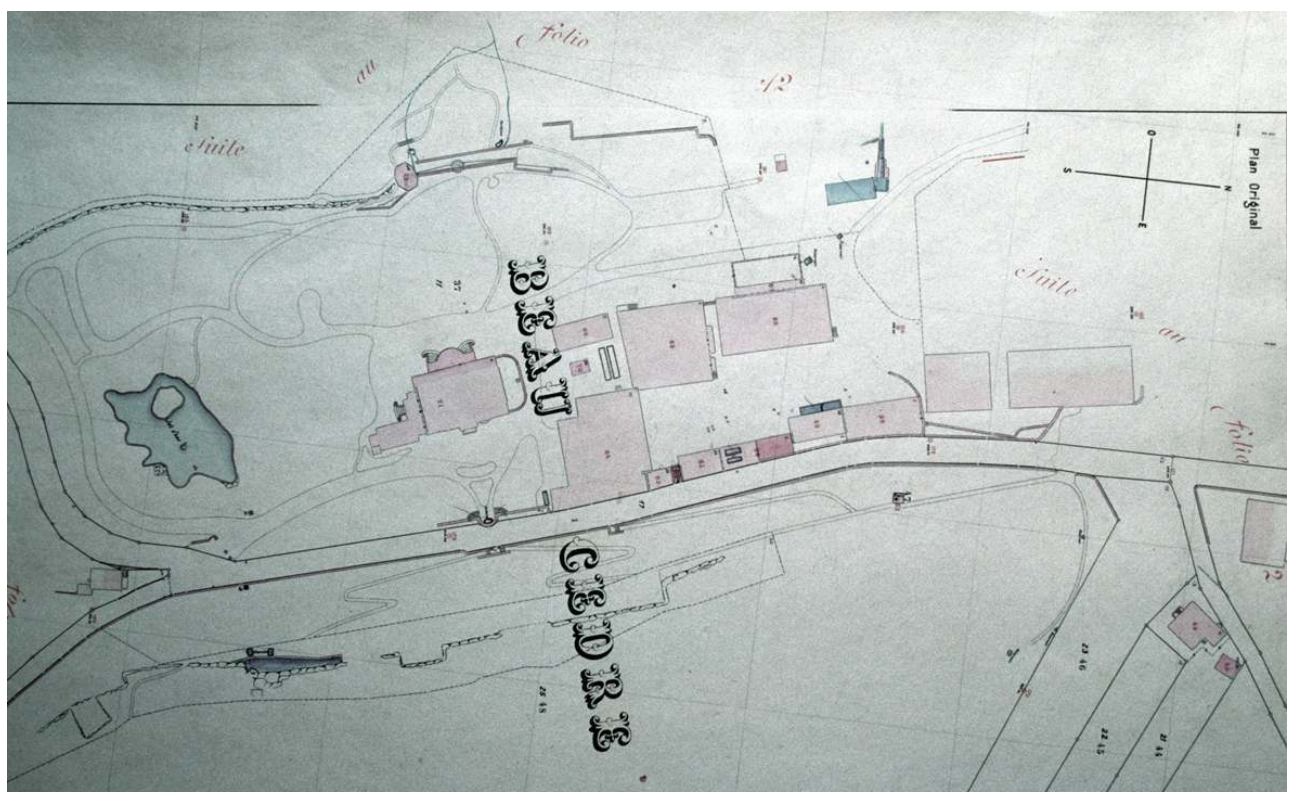

Plan cadastral de Jouxtens-Mézery, 1921-1922, folios 2 et 3 (ACV, GB 131 f).

Peu après 1883, Gustave Auberjonois publia L'album des plans et des constructions agricoles élevées en 1878 sur le domaine de Beau-Cèdre, Jouxtens près Lausanne (Suisse), à Paris, Lausanne et Londres ${ }^{3}$. Sur la couverture figure la reproduction de deux médailles gagnées respectivement à Lucerne et à Hambourg, en 1881 et 1883. Cet ouvrage contient un plan général de la ferme qui correspond pratiquement à ce qui a été réalisé (fig. $\mathbf{n}^{\circ} \mathbf{3}$ ). On y voit une douzaine d'édifices de dimensions variables, disposés autour d'une cour orientée du nord au sud et prolongée par une avenue. Le tout est desservi par un système de chemin de fer avec plaques tournantes sur lequel roulaient des wagonnets facilitant le transport des produits, du fourrage et du fumier. À l'est, un mur de clôture sépare le domaine de la rue du village qui porte aujourd'hui le nom de Beau-Cèdre. La maison de maître au sud, précédée de sa propre cour, n'est pas représentée sur ce plan.

Figure 3

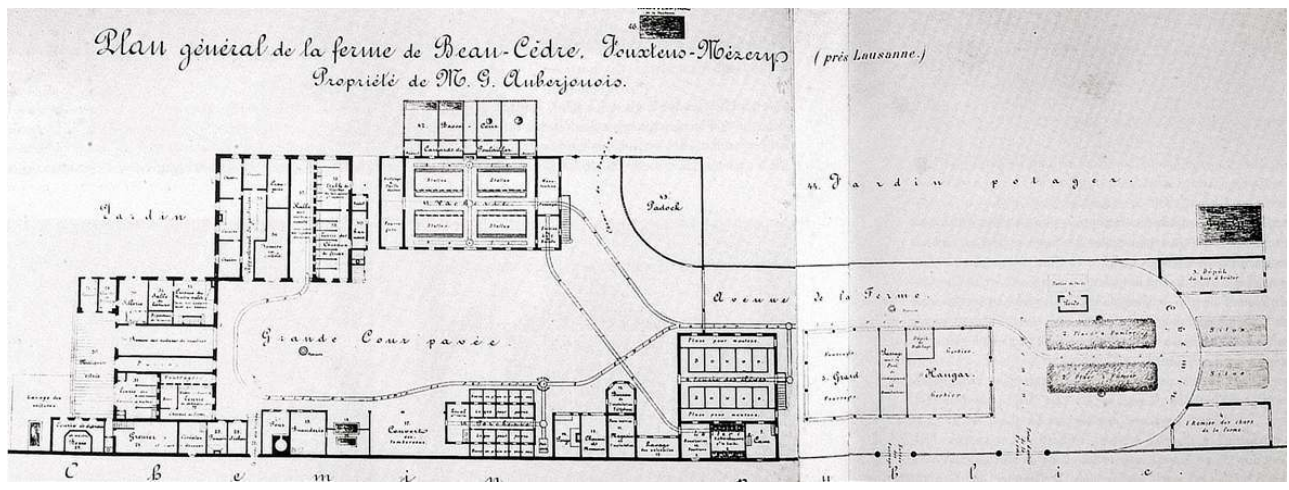

Album des plans des constructions agricoles élevées en 1878 sur le domaine de Beau-Cèdre, plan général des bâtiments de ferme (projet), vers 1883.

Collection privée. 
6 Si l'on compare cette vue d'ensemble aux planches détaillées de l'album, on constate quelques divergences ou repentirs, la laiterie ayant été bâtie à un autre emplacement que celui initialement prévu, tandis que le bureau de l'exploitation, équipé d'un téléphone, n'a pas été construit. La réalisation reste néanmoins très proche du projet publié, ce que confirme un plan cadastral levé en 1921 (voir fig. $n^{\circ} 2, n^{\circ} 3$ ) (fig. $n^{\circ} 4, n^{\circ} 5$ ).

Figure 4

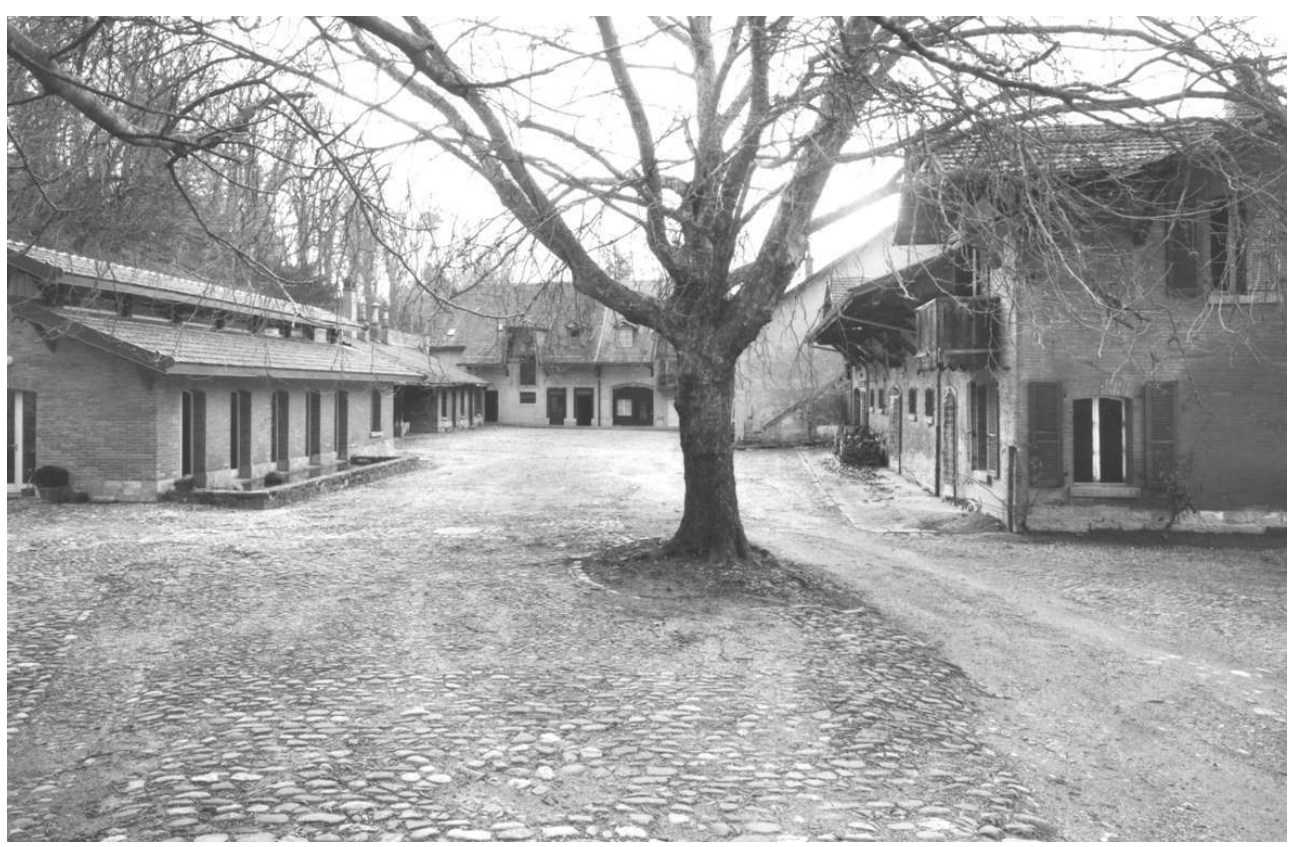

Ferme de Beau-Cèdre, à Jouxtens-Mézery, vue en direction du sud. À gauche la porcherie, au fond la ferme attestée en 1735 et à droite la vacherie.

Phot. Claude Bornand, 1999. (c) C. Bornand. 


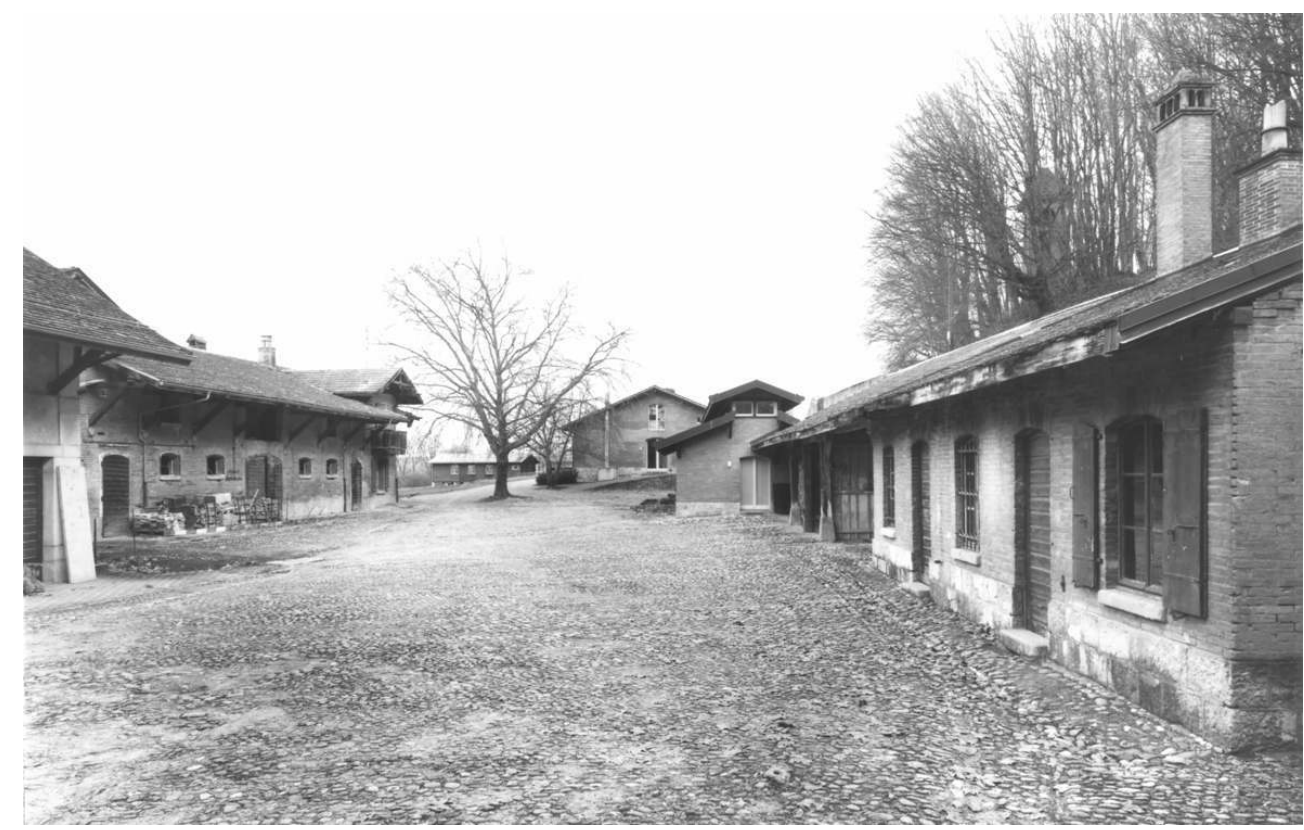

Ferme de Beau-Cèdre, vue en direction du nord. À gauche la vacherie, à droite le four et la buanderie, la porcherie et, au fond, le bâtiment des élèves.

PHOT. C. BORNAND, 1999. (C) C. BORNAND.

7 La ferme de Beau-Cèdre était principalement dévolue à la production laitière, la vacherie et la laiterie en constituant les plus beaux fleurons. Elle récoltait en outre le lait d'autres domaines de la commune et des environs. Dans la seconde moitié du XIX siècle, l'engouement pour les produits laitiers, dont la consommation était encouragée par les médecins, rendait ce marché particulièrement lucratif aux abords des villes. Ainsi, Gustave Auberjonois avait aménagé deux buvettes du lait et un point de vente dans son domaine, accessibles par un portail et un passage couvert réservé aux visiteurs. Les habitants de Lausanne s'y rendaient volontiers lors de leur promenade dominicale, afin de déguster et d'acquérir du lait frais et du beurre tout en admirant le bon fonctionnement de cette exploitation avant-gardiste. Dès 1896, et peut-être précédemment, Gustave Auberjonois disposait également d'un magasin à Lausanne pour écouler ses produits.

\section{Description des différents bâtiments : les deux dépendances du XVIII ${ }^{\mathrm{e}}$ siècle}

8 Lors de l'acquisition par Gustave Auberjonois, deux édifices du XVIII ${ }^{e}$ siècle se trouvaient sur le domaine, en plus de la maison de maître (voir fig. $\mathbf{n}^{\circ} \mathbf{1}$ ). Celui-ci les intégra à son projet de ferme modèle, sans grandes modifications, se contentant d'y apposer quelques éléments de décor et de procéder à divers réaménagements.

La dépendance qui clôt la cour du côté sud apparaît déjà sur un plan cadastral de 1735. En 1837, elle appartenait à César Constant et consistait en « un bâtiment en forte maçonnerie couvert en tuiles et bien distribué, servant pour logement des fermiers, grenier, grange et deux écuries ${ }^{4} »$. En 1860, elle fut dotée d'une grande cave voûtée surmontée d'un bûcher. 
En 1878, Gustave Auberjonois lui ajouta une sellerie et des chambres, ainsi qu'une grande marquise vitrée au sud, du côté de la cour d'honneur. Quelques ornements en bois découpés de style pittoresque et un décor de fausses briques harmonisèrent cette bâtisse aves les nouvelles constructions (fig. $\mathbf{n}^{\circ} \mathbf{6}$ ). Placée entre la cour de ferme et celle de la maison de maître, cette dépendance marque la transition entre ces deux espaces tout en assurant une communication directe grâce à un couloir médian. Selon le plan publié, elle abritait, d'ouest en est, une sellerie, une salle de lecture, l'appartement du premier valet, une remise pour les voitures des propriétaires, le passage reliant les deux cours, l'écurie des chevaux de maîtres (au sud), adossée à celle des chevaux de ferme (au nord), et une cave voûtée surmontée d'un grenier à céréales. Au sud, deux annexes enserrant la marquise vitrée servaient de remise, de chambre de nettoyage et d'écurie de débridée (actuellement sellerie). En 1888, la laiterie vint s'appuyer contre l'angle nord-est de ce bâtiment, empiétant même sur son espace. D’après ses biographes, le peintre René Auberjonois établit son premier atelier de peinture au-dessus de la sellerie.

\section{Figure 6}

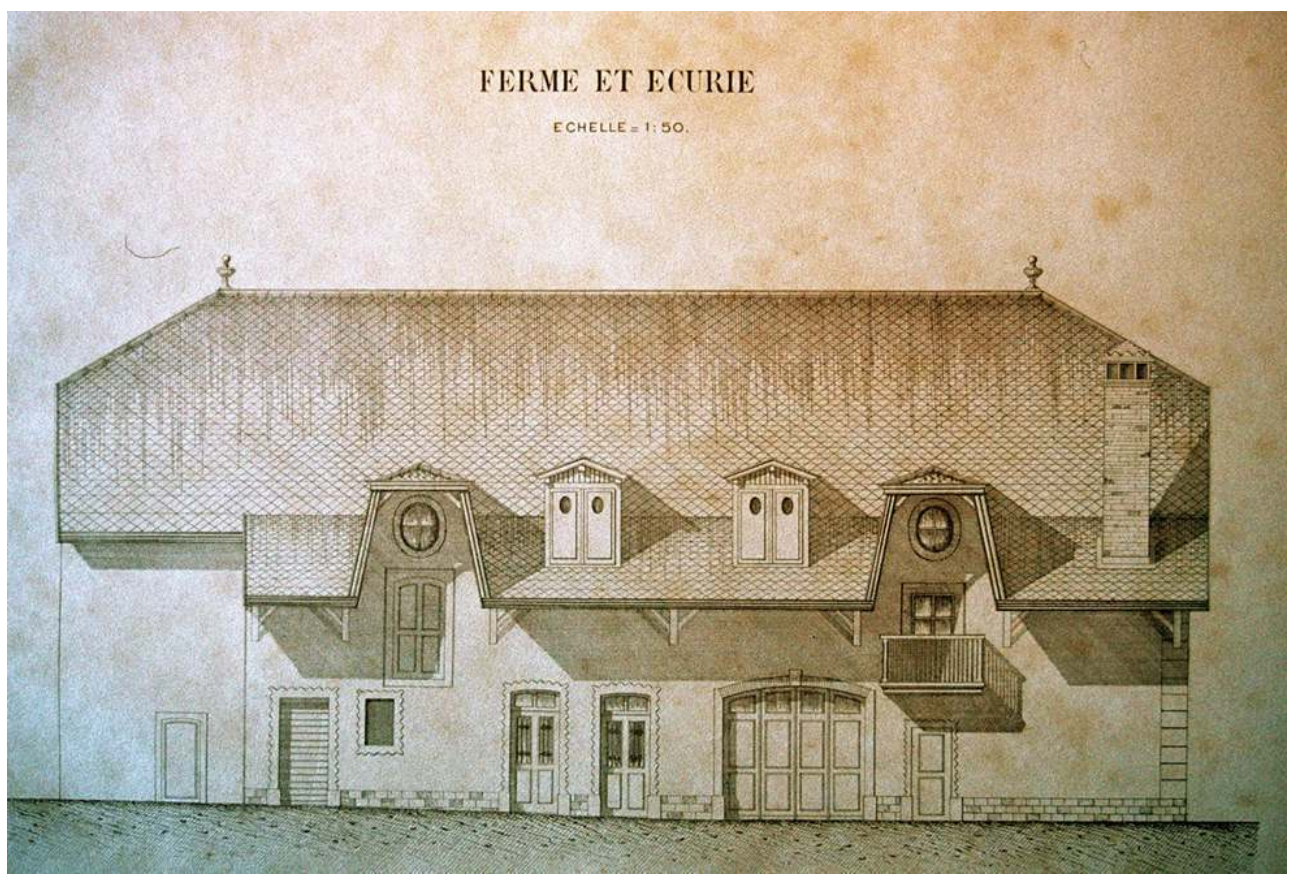

Album des plans des constructions agricoles élevées en 1878 sur le domaine de Beau-Cèdre, bâtiment de ferme antérieur à 1735, transformé au XIX siècle, façade nord.

Collection privée.

La deuxième dépendance, attestée en 1788 comme grange et écurie, fut en grande partie reconstruite en 1829-1830. En 1837, elle renfermait une grange, des écuries, une remise, une cave voûtée, une serre et un bûcher. En 1860-1862, on y créa un logement. À l'époque de Gustave Auberjonois, celui-ci était attribué au jardinier, l'ancienne grange servant de halle aux instruments et l'une des écuries étant réservée aux vaches fournissant le lait pour les enfants (fig. $\mathbf{n}^{\circ} 7$ ). 


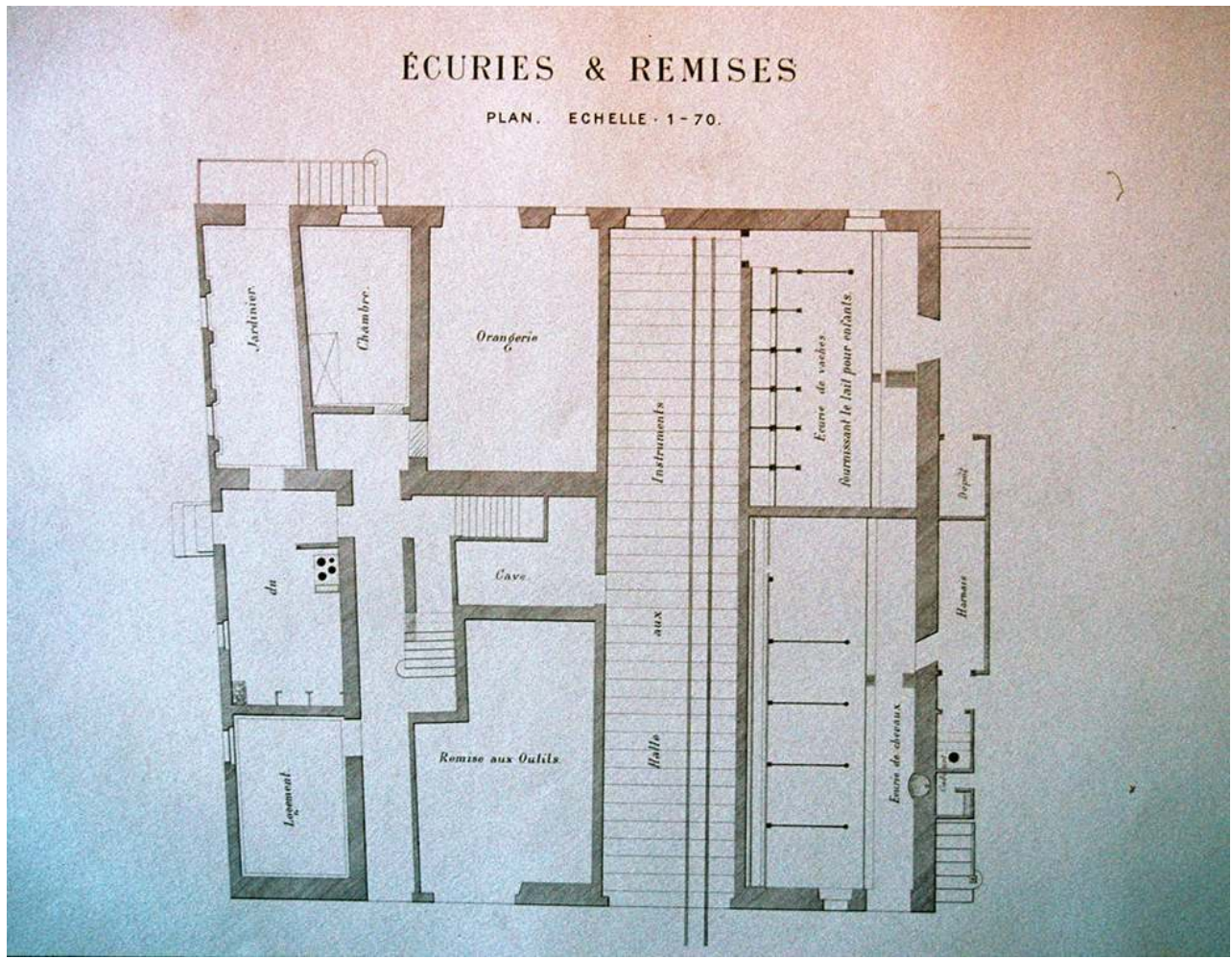

Album des plans des constructions agricoles élevées en 1878 sur le domaine de Beau-Cèdre, dépendance antérieure à 1788, transformée à diverses reprises au XIXe siècle, plan du rez-de-chaussée.

Collection privée.

\section{La vacherie}

La vacherie demeure probablement l'édifice le plus remarquable de cet ensemble (fig. $\mathbf{n}^{\circ}$ 8) (fig. $\mathbf{n}^{\circ}$ 9). Taxée en 1878 pour une valeur de 27000 francs, sa façade principale se tourne vers l'est, orientation déjà préconisée par Vitruve. Au-dessus d'un soubassement en pierres de taille, les murs de briques sont constitués de deux parements avec un vide intérieur afin d'assurer une bonne isolation. La toiture, à deux versants et faible pente, est couverte en tuiles mécaniques provenant de la tuilerie de Montchanin en Bourgogne. À cette époque, les doubles murs et les tuiles mécaniques constituent une nouveauté en Suisse romande, du moins dans le domaine de l'architecture rurale. En 1881, le Bulletin de la Société vaudoise d'agriculture considère ces murs comme excellents "contre l'humidité, la chaleur et le froid extérieur », en précisant qu'ils sont " composés de deux parpaings en carrelets de plat, laissant entre deux un vide de $0 \mathrm{~m}, 10$ environ ${ }^{5} »$. 
Figure 8

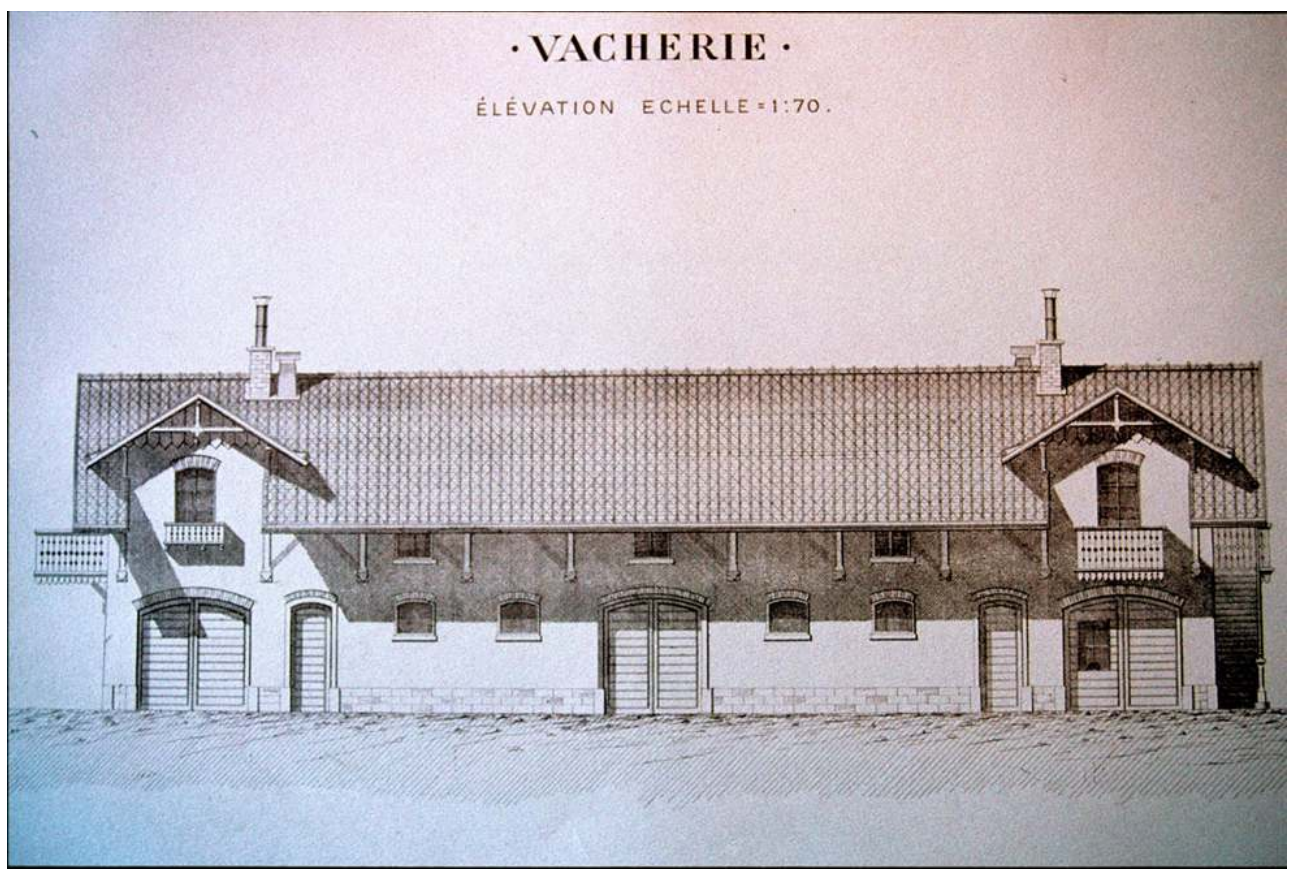

Album des plans des constructions agricoles élevées en 1878 sur le domaine de Beau-Cèdre, la vacherie, façade principale, à l'est.

Collection privée.

Figure 9

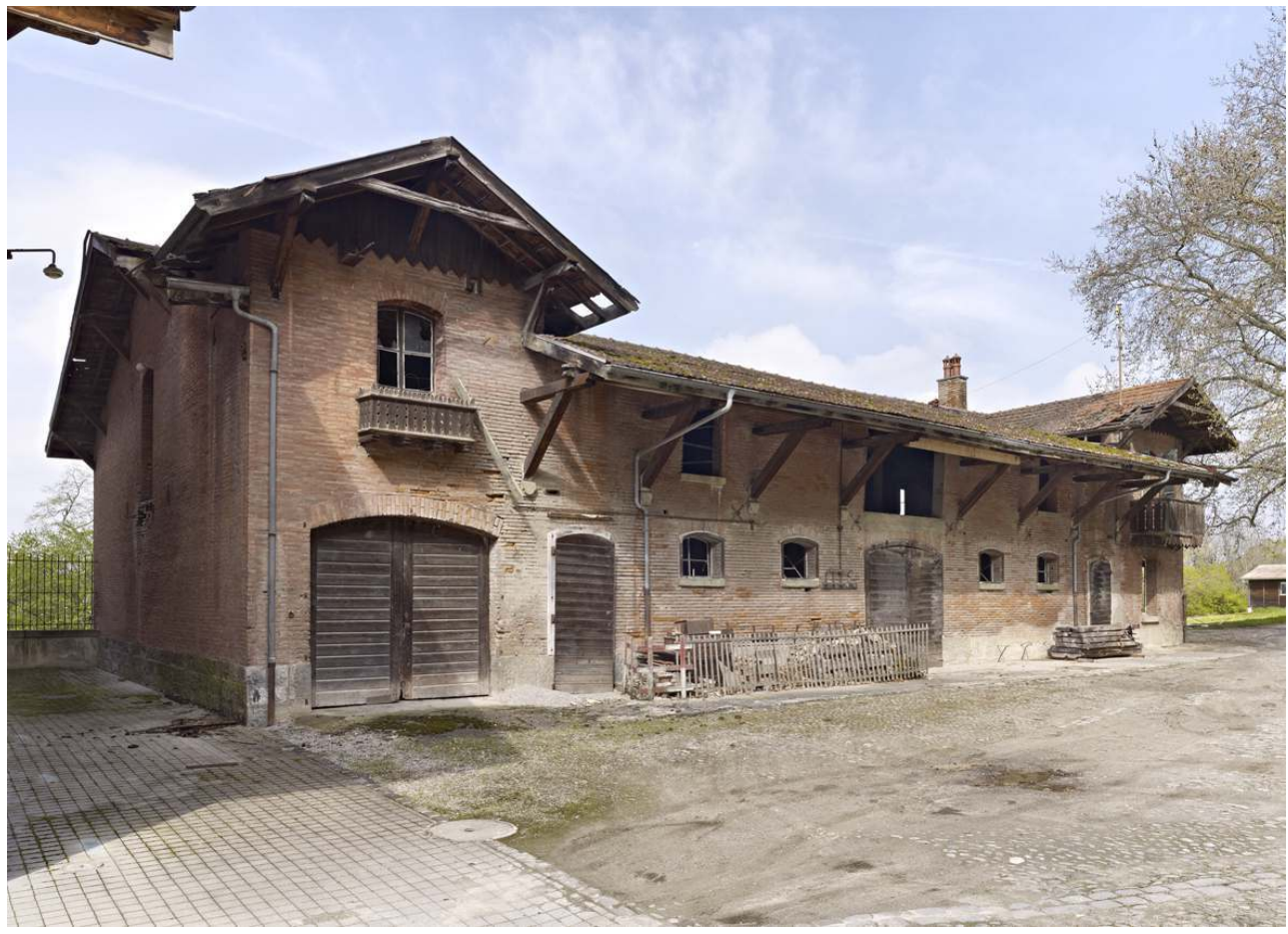

Ferme de Beau-Cèdre, la vacherie, vue de la façade principale.

Phot. R. Gindroz, 2012. (c) R. Gindroz. 
12 Le rez-de-chaussée de la vacherie abritait une grande étable longitudinale destinée à 28 vaches, flanquée de locaux annexes réservés au stockage et à la manutention, ainsi qu'une buvette du lait. Cette étable était divisée en quatre compartiments, des rails quadrillant l'espace avec des plaques tournantes aux croisements (fig. $\mathbf{n}^{\circ} \mathbf{1 0}$ ). Ces dernières, conservées, portent la marque d'une entreprise parisienne (fig. $\mathbf{n}^{\circ} \mathbf{1 1}$ ).

Figure 10

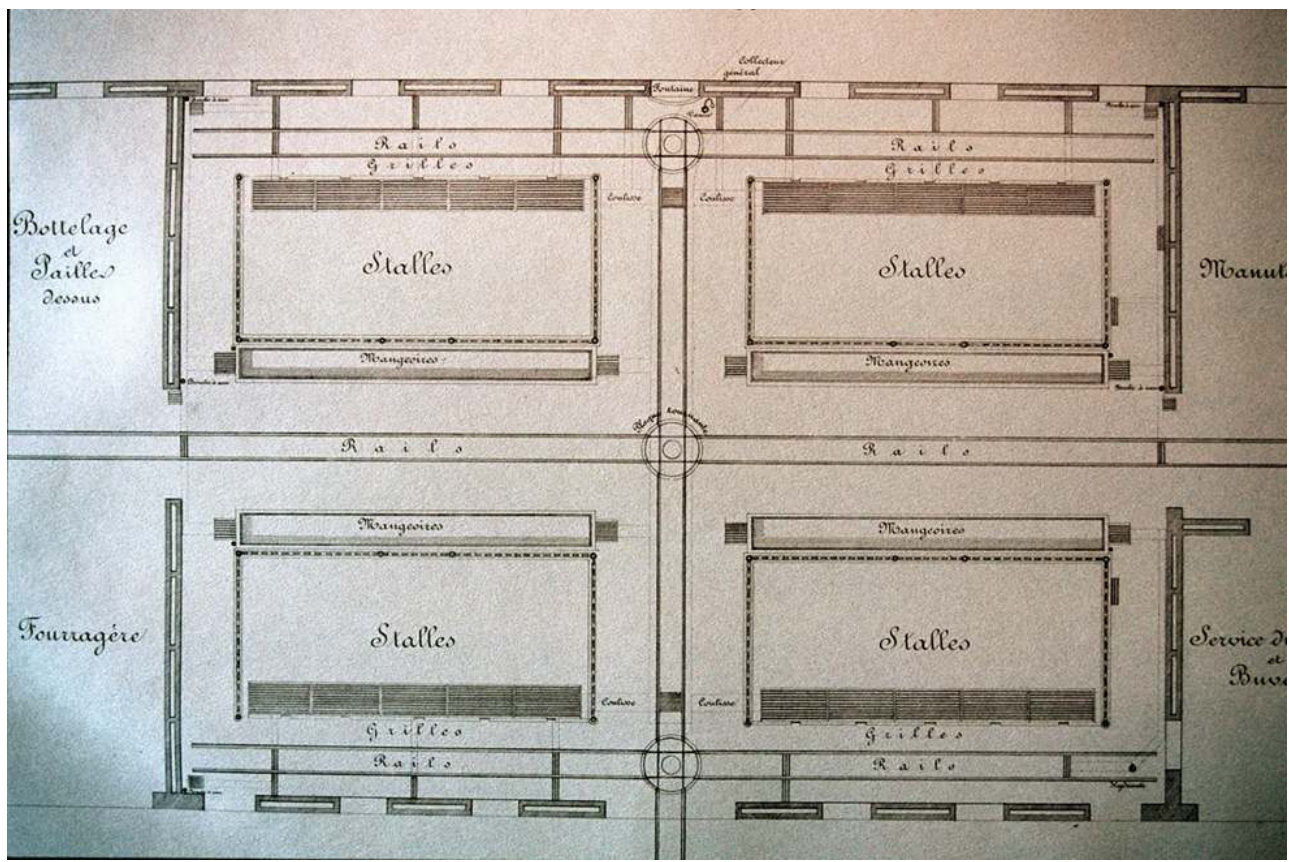

Album des plans des constructions agricoles élevées en 1878 sur le domaine de Beau-Cèdre, la vacherie, plan de l'étable au rez-de-chaussée.

Collection privée. 


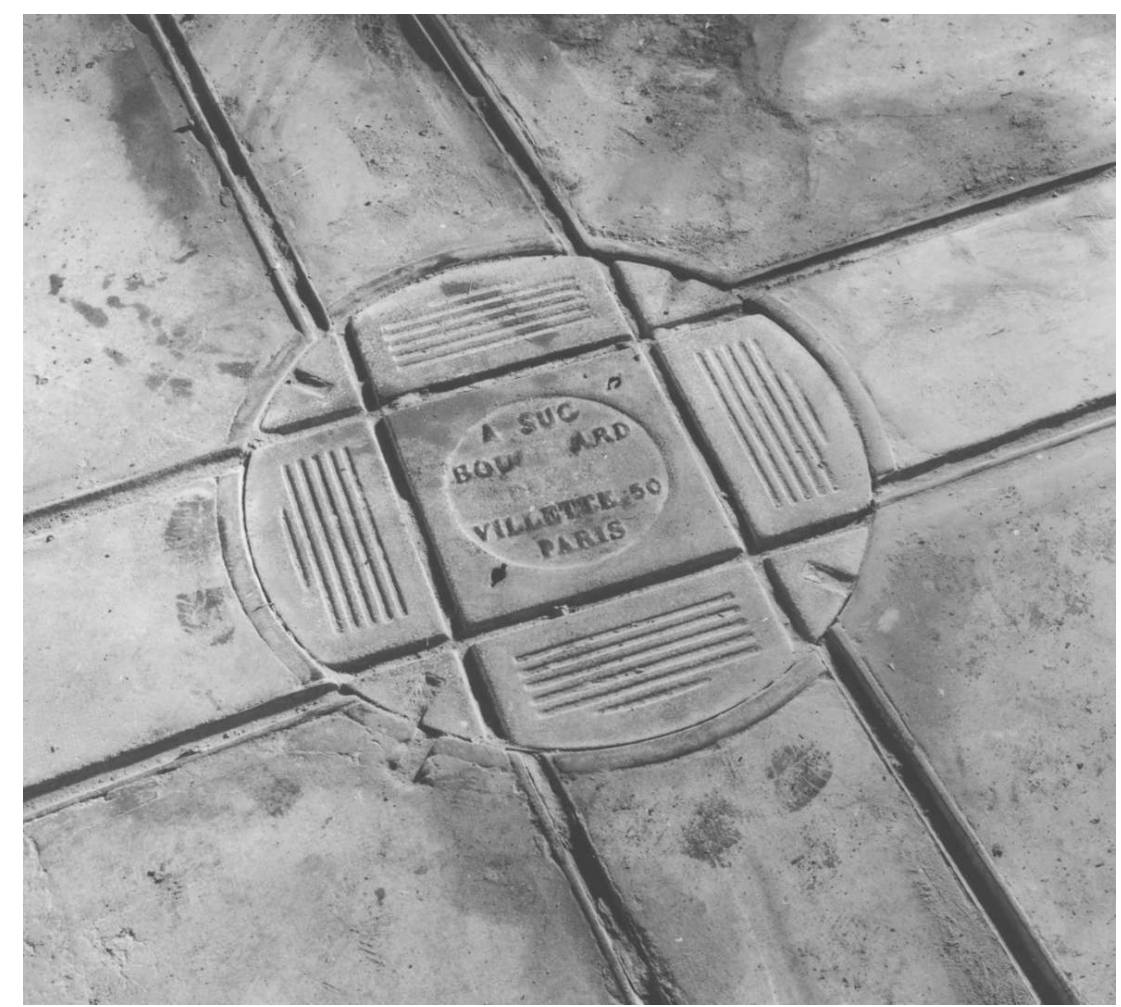

Vacherie de Beau-Cèdre, intérieur de l'étable, plaque tournante du chemin de fer avec l'inscription: Asuc, Boulevard de la Villette 50, Paris.

Phot. C. Bornand, 1999. (c) C. Bornand.

Les vaches se faisaient face de part et d'autre d'un couloir central d'alimentation dans lequel se trouvaient les mangeoires. Une claire-voie dotée d'une ouverture pour chaque bête permettait à ces dernières d'accéder ou non à leur crèche (fig. $\left.\mathbf{n}^{\circ} \mathbf{1 2}\right)$ (fig. $\left.\mathbf{n}^{\circ} \mathbf{1 3}\right)$. Ce système, nommé "cornadie » ou "cornadise », appliqué depuis des décennies dans certaines régions d'Allemagne ou de France, est longuement décrit dans le traité de Louis Bouchard-Huzard ${ }^{6}$. Inconnu alors dans le canton de Vaud, il fut perfectionné par Gustave Auberjonois qui alla jusqu'à déposer un brevet pour la serrure du volet de ses « cornadises ». La presse s'en fit rapidement l'écho, comme en témoigne un texte paru en 1881 dans le Bulletin de la Société vaudoise d'agriculture : "À Beau-Cèdre, le transport des denrées alimentaires se fait par des wagonnets roulant sur des chemin de fer avec plaques tournantes et tous les perfectionnements de l'industrie moderne; chacun des compartiments de l'étable peut contenir 3 [en réalité 7] vaches, la tête tournée contre une paroi à claire-voie qui prend le nom de cornadise et sépare ce compartiment du corridor central. Cette cornadise est percée d'un guichet devant la place occupée par chaque animal; ce guichet, de 0m,40 sur 0m,60 s'ouvre à la hauteur de la crèche pour l'heure des repas. [...] L'expérience a démontré que quelle que soit la longueur de ses cornes, la vache s'habitue très vite à passer la tête à travers la baie ouverte devant elle et qu'elle l'y laisse pendant tout le temps où elle mange, évitant ainsi de jeter sous ses pieds les portions de fourrage que sa friandise dédaigne. [...] Un robinet placé à l'extrémité de la crèche amène l'eau d'abreuvage ou de propreté; à Beau-Cèdre cette eau est présentée successivement devant chaque vache dans une petite auge roulant sur les bords de la crèche, couverts à cet effet de deux bandes de fer d'angle ${ }^{7}$. » On précise encore que chaque vache dispose d'une largeur de 
crèche de 1,25 $\mathrm{m}$ et d'une hauteur de $3 \mathrm{~m}$, dimensions préconisées par Louis BouchardHuzard et d'autres agronomes 8 .

Figure 12

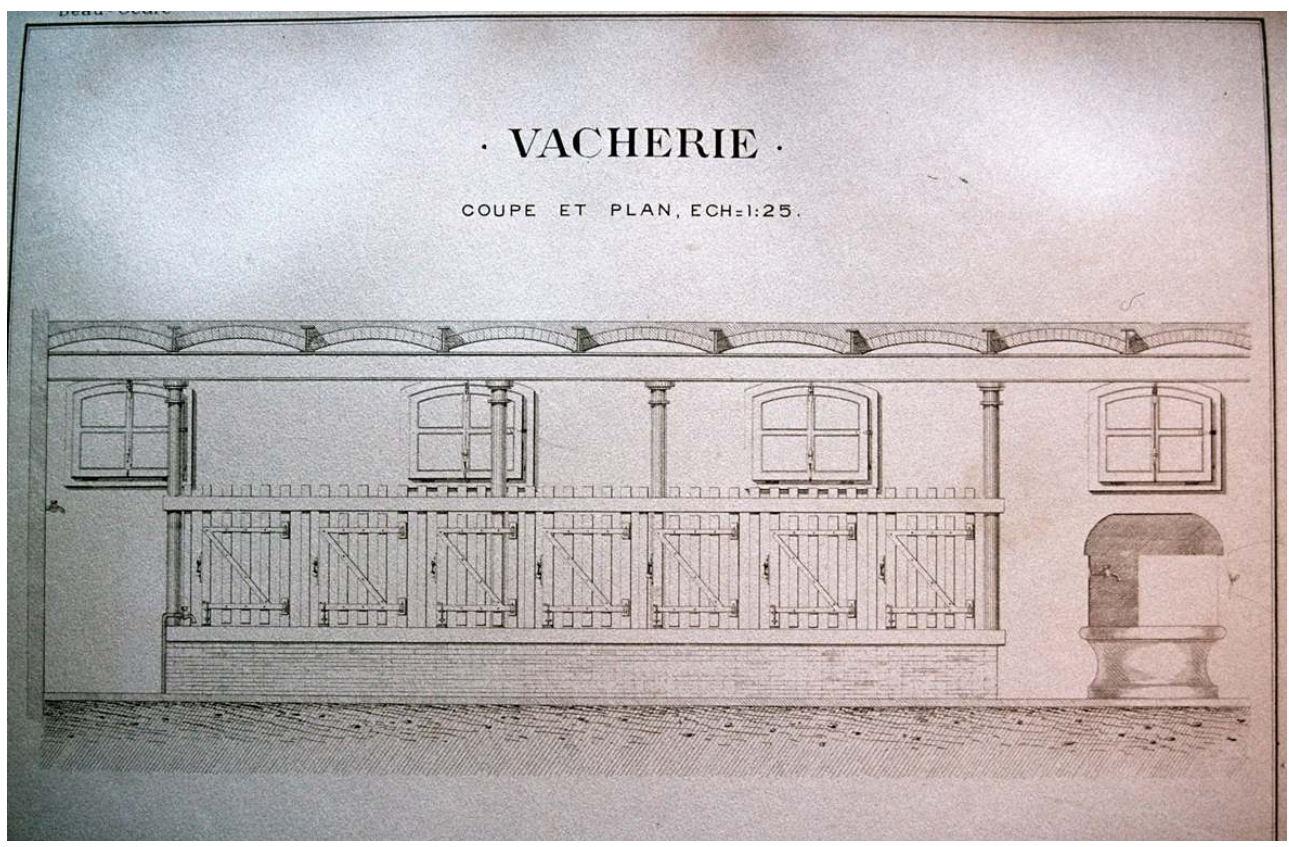

Album des plans des constructions agricoles élevées en 1878 sur le domaine de Beau-Cèdre, la vacherie coupe longitudinale partielle de l'étable, avec les «cornadises » séparant les bêtes des mangeoires. Collection privée. 
Figure 13

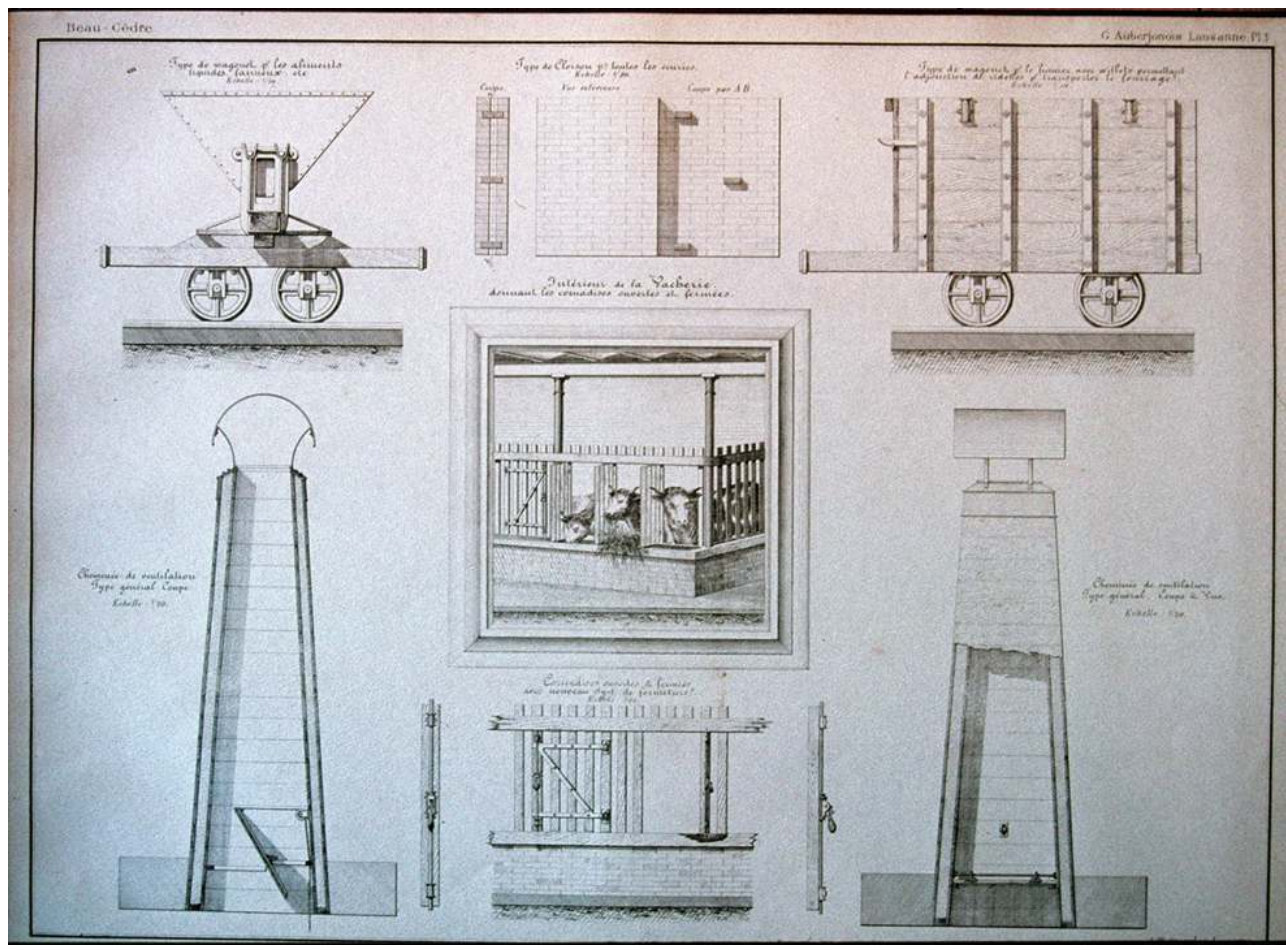

Album des plans des constructions agricoles élevées en 1878 sur le domaine de Beau-Cèdre, la vacherie, planche représentant les wagonnets utilisés pour transporter les aliments, le fumier et le fourrage, le système de double mur, les cheminées de ventilation et les « cornadises » ouvertes et fermées.

Collection privée.

Derrière la couche des vaches, des grilles surmontaient des canaux souterrains d'évacuation du lisier, inondables grâce à des hydrantes placées à l'intérieur de l'étable ou par une fontaine alimentée par un conduit d'eau sous pression (voir fig. $\left.\mathbf{n}^{\circ} \mathbf{1 0}, \mathbf{n}^{\circ} \mathbf{1 2}\right)$ (fig. $\left.n^{\circ} 14\right)$. 


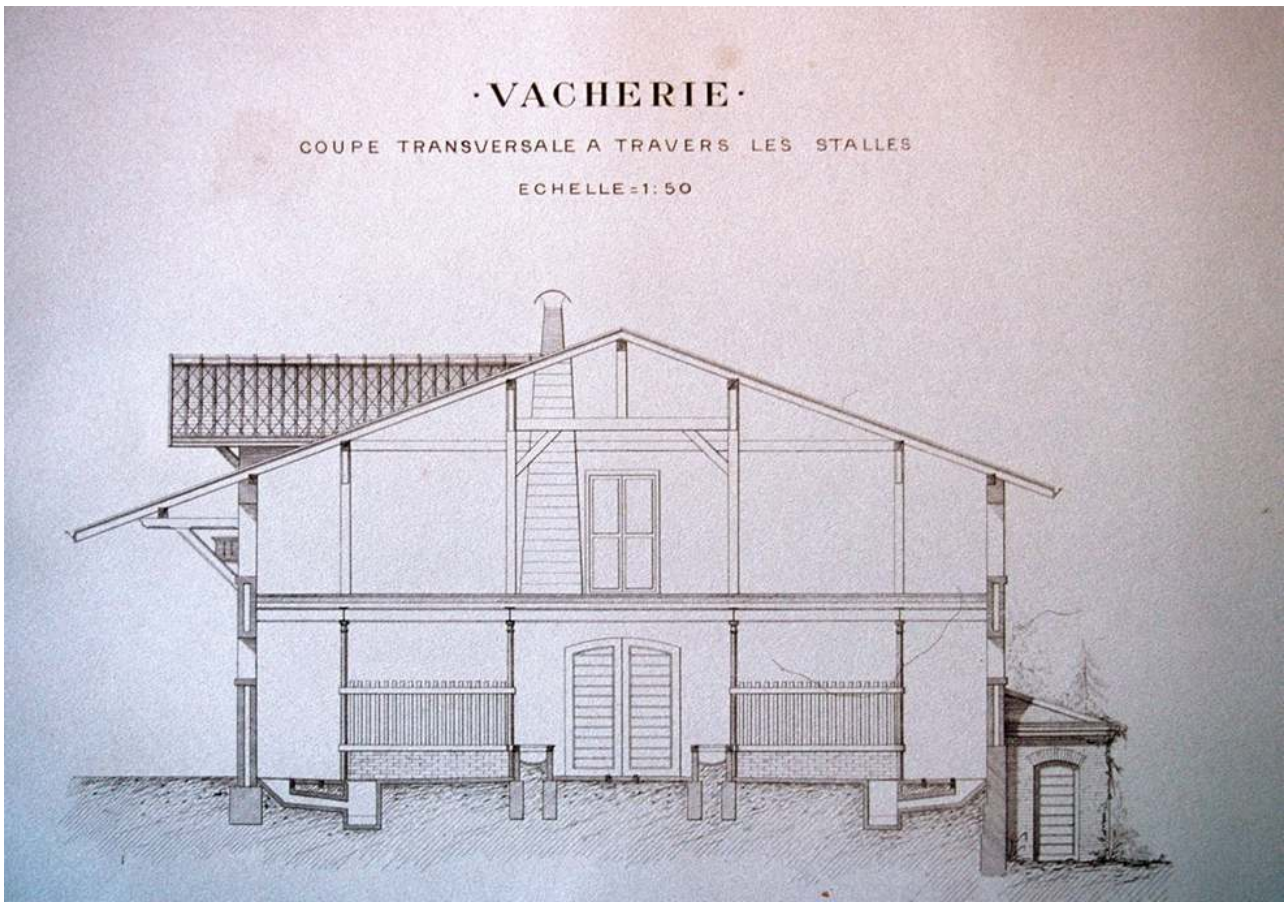

Album des plans des constructions agricoles élevées en 1878 sur le domaine de Beau-Cèdre, la vacherie, coupe transversale, avec les « cornadises », l'une des cheminées de ventilation et les canaux d'évacuation du lisier inondables grâce à des hydrantes.

Collection privée.

L'intérieur de l'étable, partiellement préservé jusqu'en 2013, demeure très ouvert. Le plafond est constitué de poutrelles en $\mathrm{H}$ à entrevous en berceau segmentaire en brique reposant sur de grandes poutres en fonte soutenues par des colonnettes dans ce même matériau (voir fig. $\left.\mathbf{n}^{\circ} 12\right)$ (fig. $\mathbf{n}^{\circ}{ }^{15}$ ). Ce mode de bâtir, influencé par l'architecture industrielle, est à cette époque particulièrement avant-gardiste en milieu rural, du moins en Suisse romande. De même, les huisseries des fenêtres oblongues, à châssis basculant, constituent une nouveauté dans cette région où dominent les baies verticales à deux battants (voir fig. $\mathbf{n}^{\circ} \mathbf{1 2}$ et $\mathbf{n}^{\circ} \mathbf{1 5}$ ). Ces ouvertures, vantées par Louis Bouchard-Huzard, apportent une lumière plus uniforme à l'intérieur de l'étable, tout en envoyant l'air froid vers le haut, ce qui lui laisse le temps de se réchauffer avant d'atteindre les bêtes'. À Beau-Cèdre, deux types de châssis étaient utilisés alternativement : le premier vitré en hiver, le second, garni d'un treillis, en été. Finalement, de grandes cheminées de ventilation de forme pyramidale, préconisées par les agronomes depuis pratiquement un siècle, assainissaient l'air de l'étable. 


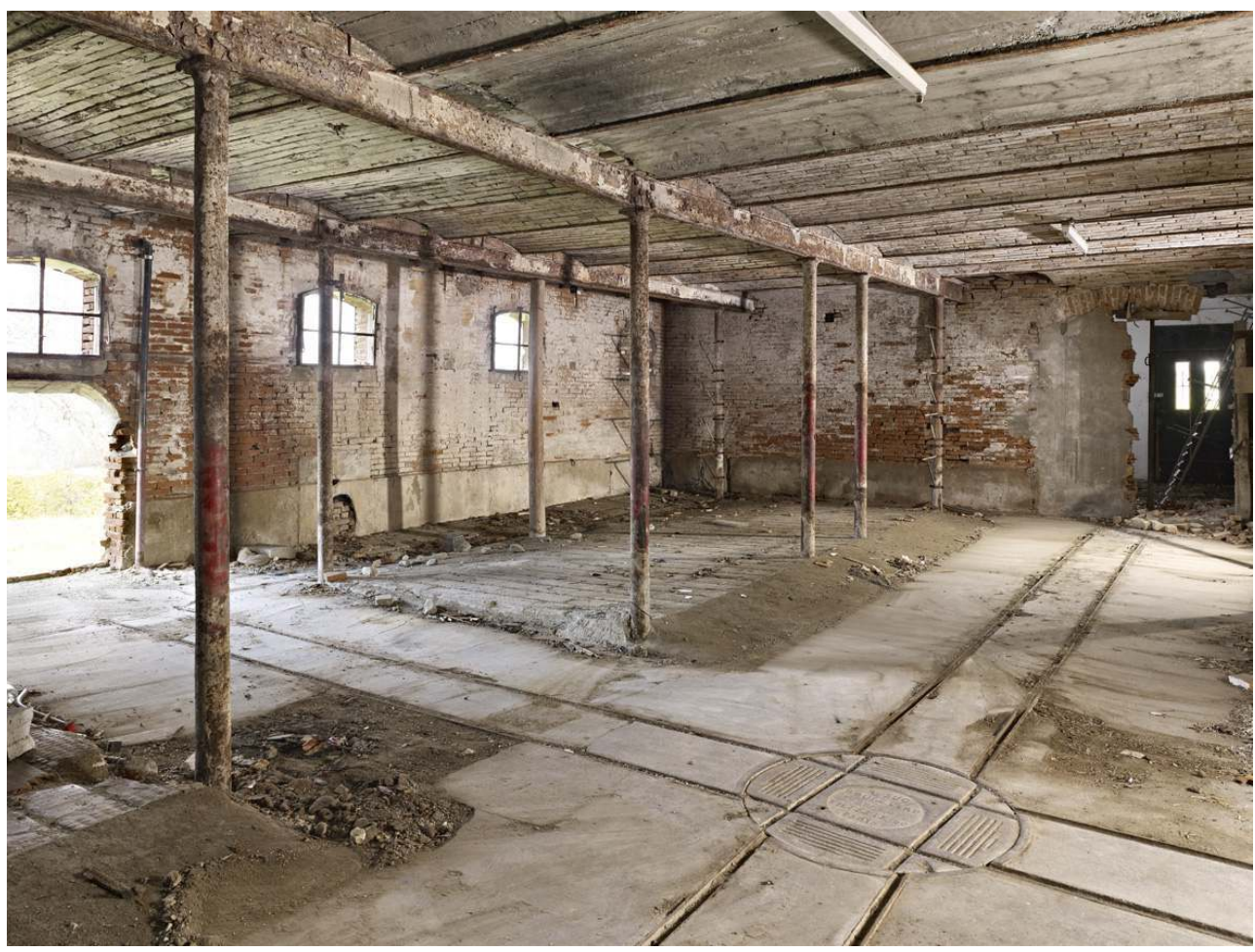

Ferme de Beau-Cèdre, intérieur de la vacherie.

Phot. R. Gindroz, 2012. (c) R. Gindroz.

16 L'étage de la vacherie, sous le comble, renfermait les chambres des vachers au sud, celle du premier vacher disposant d'un regard s'ouvrant sur l'étable pour surveiller les bêtes. L'espace central contenait un grand dépôt, tandis qu'au nord, se trouvaient une pharmacie et le bureau de la direction, doté d'un balcon côté cour pour s'assurer de la bonne marche du domaine (voir fig. $\left.\mathbf{n}^{\circ} \mathbf{8}\right)$.

La vacherie de Beau-Cèdre tranchait ainsi radicalement avec les constructions rurales environnantes, tant par ses dispositions et ses équipements que par les matériaux utilisés. Le plan, subdivisé en quatre compartiments avec un couloir central d'alimentation et un réseau de chemin de fer, s'inspire visiblement de celui de la ferme impériale de Vincennes, bâtie en 1859 sur l'ordre de Napoléon III par Tisserand et Ronsier. Plusieurs relevés de cet ensemble sont publiés dans le traité de Louis Bouchard-Huzard ${ }^{10}$, que Gustave Auberjonois possédait certainement, sans compter ses propres séjours parisiens.

\section{La laiterie et la buvette du lait}

18 En 1888, une laiterie considérée comme «modèle » est bâtie dans l'angle sud-est de la cour et en empiétant dans la volumétrie de l'ancienne ferme attestée en 1735. Elle disposait d'un système perfectionné pour remplir les bouteilles de lait, les transporter et les laver, d'une baratte à beurre, d'un malaxeur, d'une écrémeuse Laval et d'une chaudière à vapeur (fig. $\left.\mathbf{n}^{\circ} \mathbf{1 6}\right)\left(\mathbf{f i g} \mathbf{n}^{\circ}{ }^{17}\right)$. La production se concentrait sur la fabrication de la crème et du beurre, ainsi que sur la mise en bouteille du lait frais. L'ensemble se décomposait en plusieurs unités : la laiterie contenant les baquets pour refroidir le lait, précédée de la buvette, la cave voûtée et le lieu de transformation du lait. Ce dernier, 
comprenant l'espace de la beurrerie et celui de la mise en bouteilles, communiquait avec un petit magasin qui jouxtait le local de la chaudière à vapeur.

Figure 16

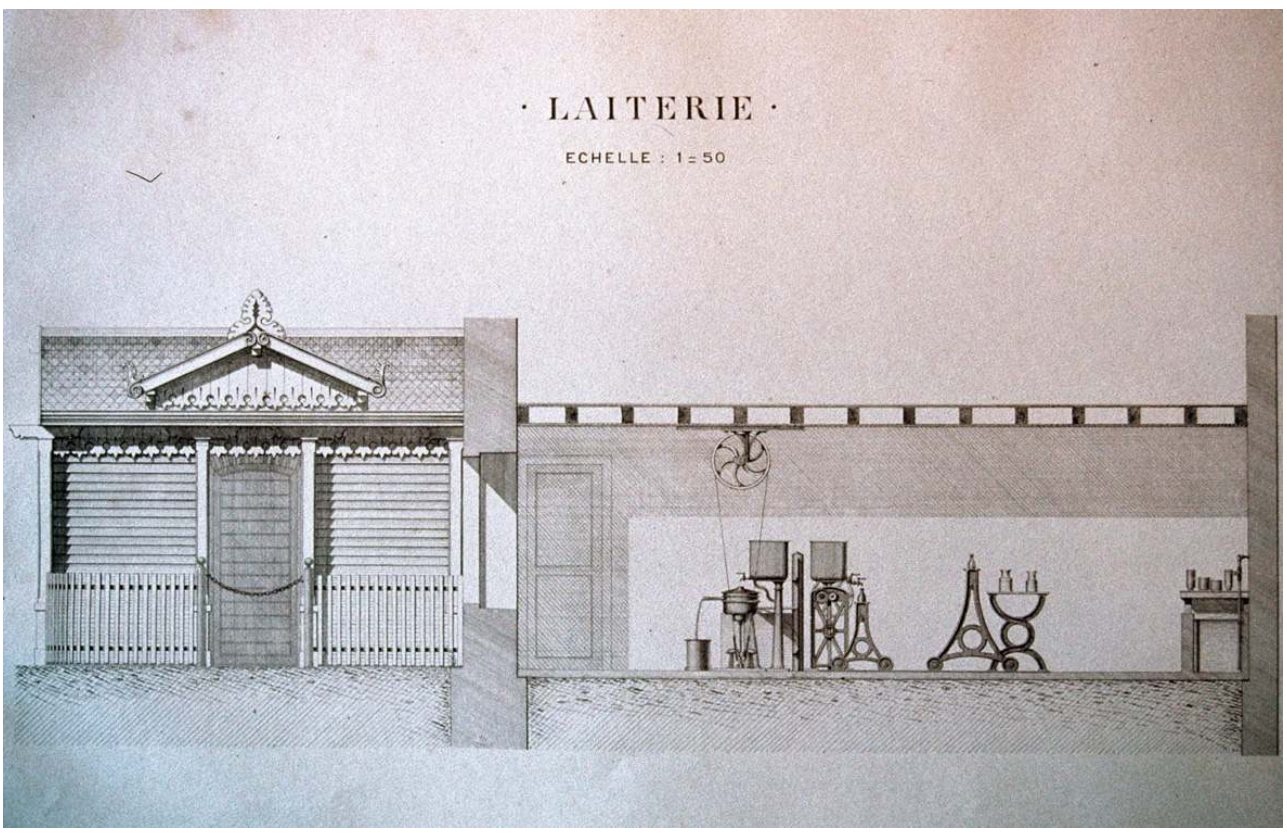

Album des plans des constructions agricoles élevées en 1878 sur le domaine de Beau-Cèdre, la laiterie et son équipement moderne, avec la buvette du lait sur la gauche.

Collection privée. 


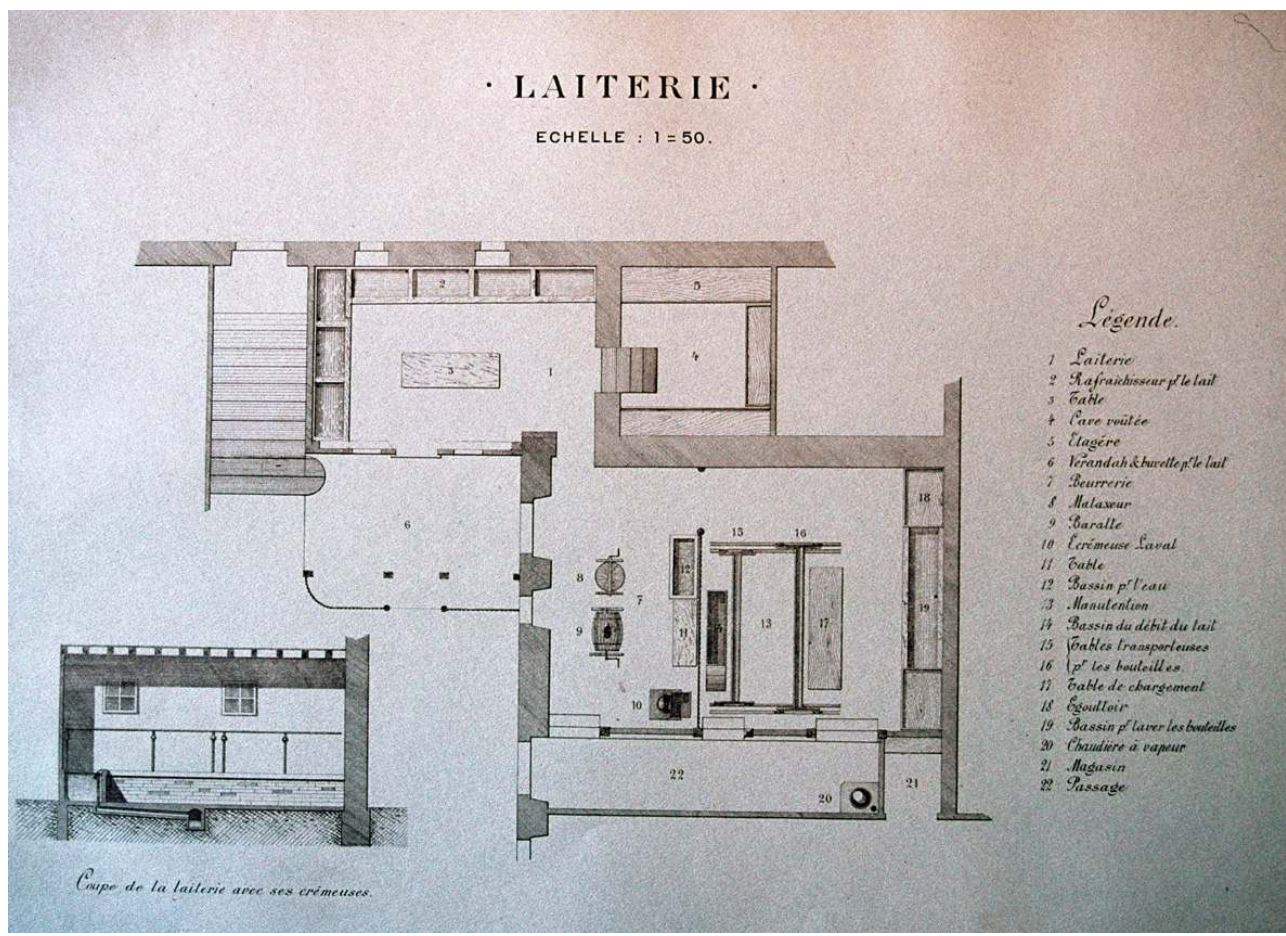

Album des plans des constructions agricoles élevées en 1878 sur le domaine de Beau-Cèdre, plan et coupe de la laiterie.

Collection privée.

Fleuron de Beau-Cèdre, cette laiterie fit l'objet de la plus grande attention de la part de Gustave Auberjonois qui ne cessa de l'améliorer. En mars 1889, il se rendit à l'Exposition universelle de Paris afin de se renseigner à ce propos, comme en témoigne, sans grand enthousiasme, son fils René : «L'après-midi, nous avons erré de laiterie en laiterie, et j'étais tellement fatigué que je m'endormais sur les bancs. Papa liait connaissance avec tous les grands laitiers et discutait longuement ${ }^{11} »$. Une dizaine d'années plus tard, son second fils, Maurice, en visite à l'Exposition internationale d'agriculture à Exeter, livre un commentaire plus élogieux : «Particulièrement intéressante la working dairy, avec le radiateur dans lequel on verse du lait et l'on recueille du beurre à l'autre bout. Également les centrifuge[use]s Laval, toujours au premier rang [...]. Je pense combien papa serait intéressé par tout ce qu'on voit ici et regrette bien qu'il ne soit pas avec moi ${ }^{12}$ ».

$\mathrm{Au}$ nord de la laiterie, un escalier et un passage couvert permettaient aux visiteurs d'accéder à la propriété. Au-delà s'étend le bâtiment du four et de la buanderie, jouxtant une fontaine couverte dotée de deux bassins distincts, l'un servant à laver le linge, l'autre

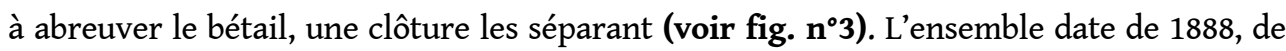
même que la remise à tombereaux adjacente.

\section{La porcherie}


Figure 18

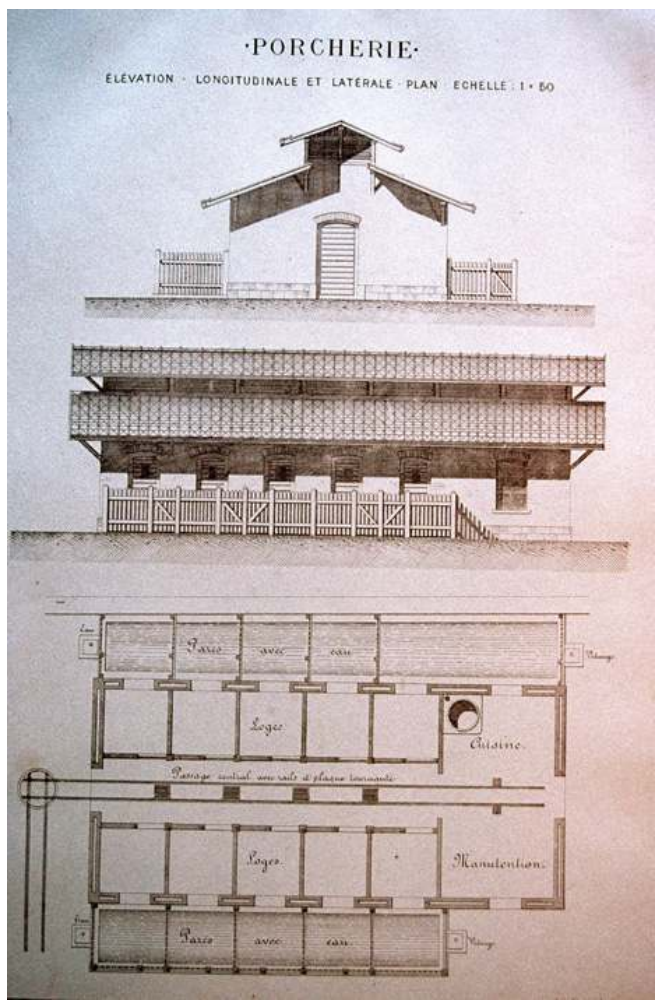

Album des plans des constructions agricoles élevées en 1878 sur le domaine de Beau-Cèdre, la porcherie élévations (façades sud et ouest) et plan avec les loges s'ouvrant sur des enclos extérieurs.

Collection privée.

\section{Figure 19}

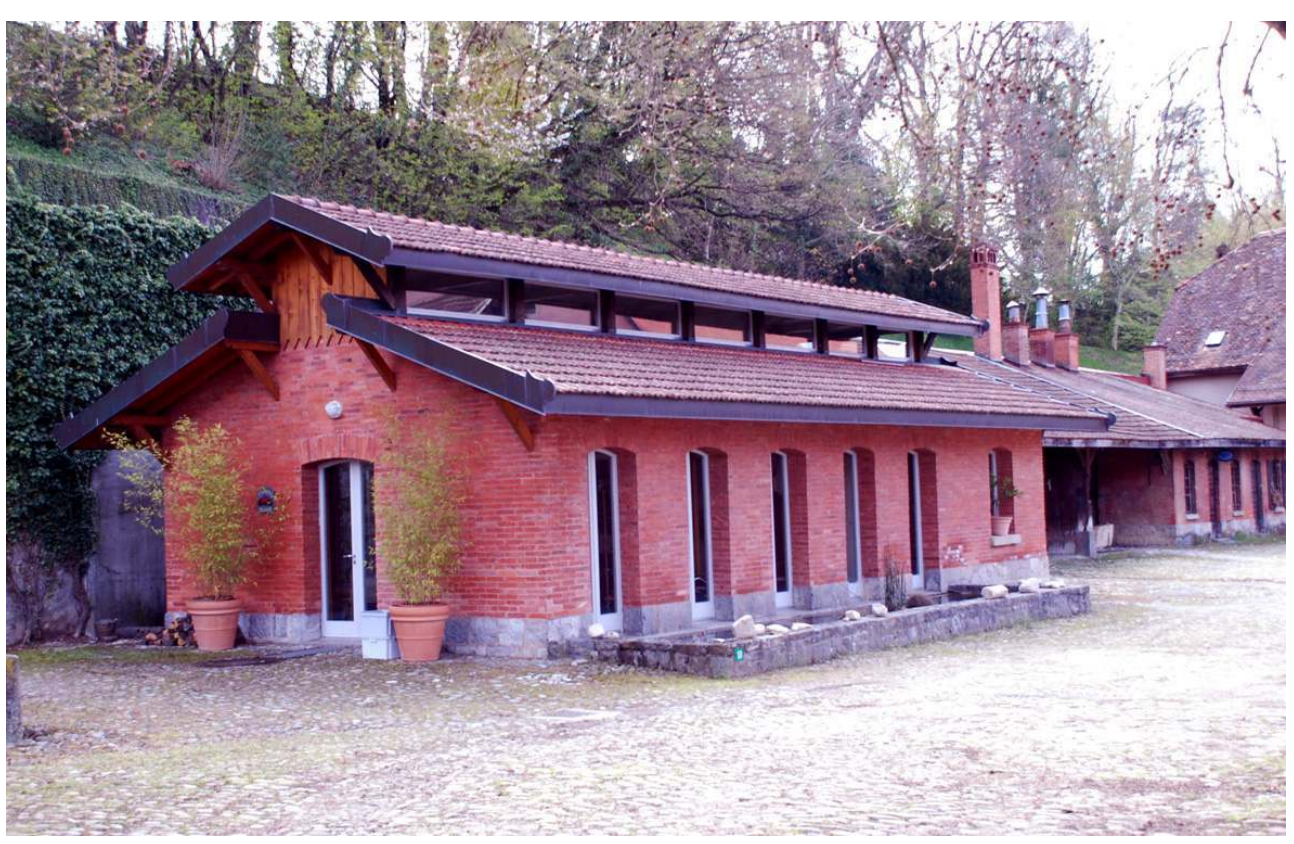

Ferme de Beau-Cèdre, la porcherie transformée en bureau d'architecture.

Phot. I. Roland, 2008. (C) I. Roland. 
La porcherie, actuellement transformée en bureau d'architecture, constitue également l'une des pièces maîtresses de Beau-Cèdre, malgré ses dimensions modestes (fig. $\mathbf{n}^{\circ} \mathbf{1 8}$ ) (fig. no19). Gustave Auberjonois y élevait des porcs du Yorkshire dont il était particulièrement fier et qui obtinrent plusieurs médailles aux concours agricoles. L'édifice, taxé en 1888, comprend un niveau bâti en doubles murs de briques sur un soubassement en pierres. Il abritait une dizaine de bêtes dans des loges individuelles réparties de part et d'autre d'un couloir central d'alimentation desservi par des rails (voir fig. $\mathbf{n}^{\circ}$ 18). Chaque loge disposait d'un petit enclos extérieur dont le fond pouvait être inondé afin que les porcs s'ébattent dans l'eau. Le sol était constitué d'un plancher à claire-voie placé au-dessus d'une fosse s'inclinant vers un canal central d'évacuation du lisier (fig. $\mathbf{n}^{\circ} \mathbf{2 0}$ ). Les auges placées à cheval entre les loges et le couloir d'alimentation, étaient dotées d'un couvercle curviligne basculant, permettant de les remplir ou de les nettoyer sans se faire mordre. Une cuisine équipée d'une chaudière et un local de manutention occupaient la première travée au sud du bâtiment.

Figure 20

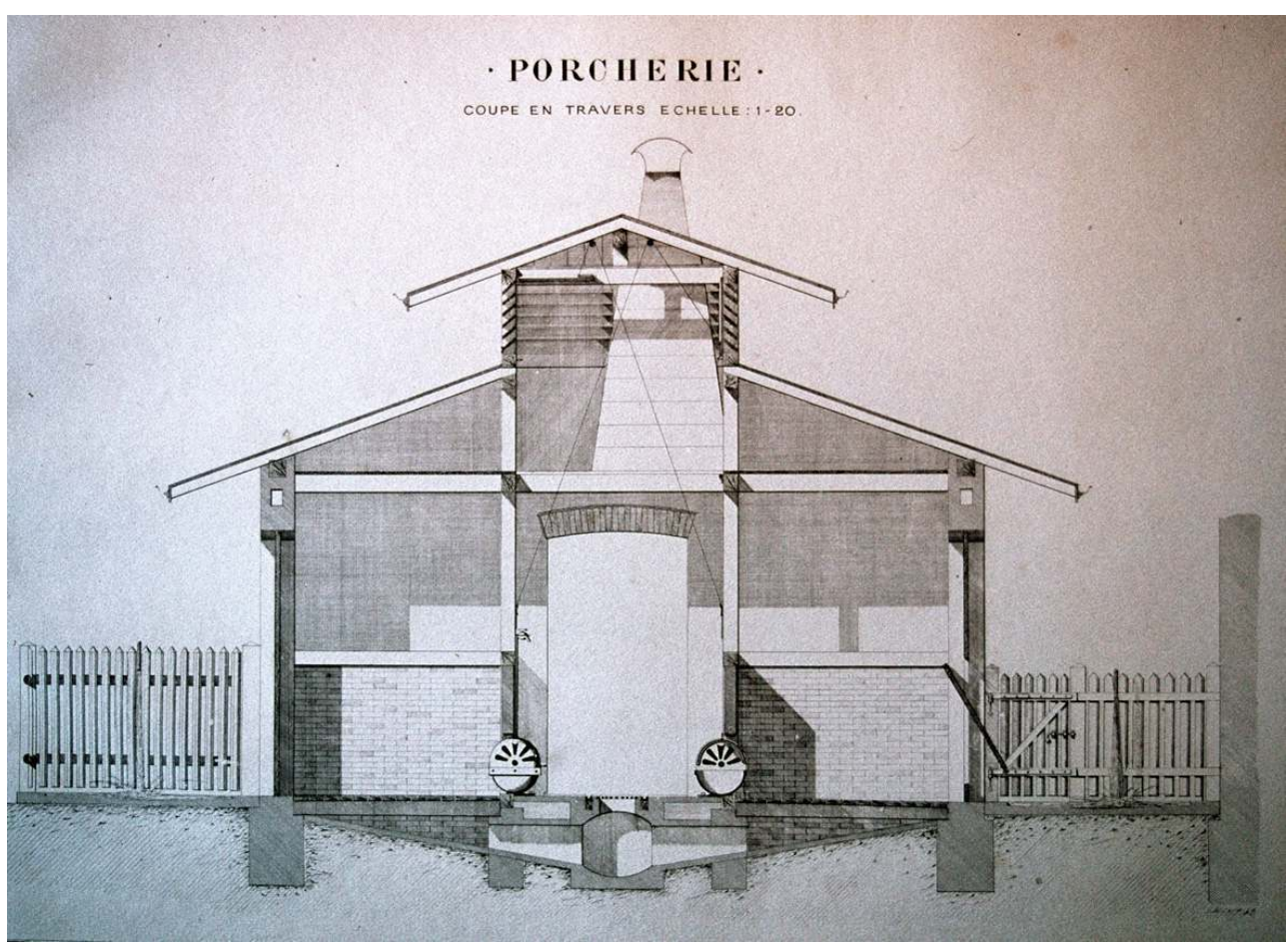

Album des plans des constructions agricoles élevées en 1878 sur le domaine de Beau-Cèdre, la porcherie, coupe transversale, avec les persiennes mobiles, les mangeoires à couvercle basculant et les canaux d'évacuation.

Collection privée. 
Un hangar et une forge, datant de 1888, s'élevaient au nord de la porcherie. Encore dessinés sur le plan cadastral de 1921-22, ils ont été démolis par la suite.

\section{Le bâtiment des élèves}

Un bâtiment dit « des élèves », destiné au jeune bétail, fut taxé en 1877 pour une valeur de 10000 francs. De plan rectangulaire avec des doubles murs en brique, il comprend un niveau sous une toiture à deux pans en tuiles mécaniques (fig. $\mathbf{n}^{\circ} \mathbf{2 1}$ ). L'espace intérieur était subdivisé en cinq boxes disposés de part et d'autre d'un couloir d'alimentation desservi par un chemin de fer. À l'arrière de ces enclos s'étendait une place pour les jeunes veaux et les agneaux. Le plafond était formé de "murettes à l'italienne », c'est-àdire de "poutres garnies, entre-deux, de mortier bâtard composés de chaux, de sable et de gypse ", selon la définition du Bulletin de la Société vaudoise d'agriculture, qui précise que ce procédé n'a pas réussi à la campagne de $\mathrm{M}$. Auberjonois, «mais peut-être a-t-on négligé les précautions indispensables, le choix des bois et l'enduit hydrofuge ${ }^{14}{ }$. Dans tous les cas, le plafond d'origine fut finalement remplacé par des poutrelles en $\mathrm{H}$ avec des hourdis en briques creuses de fabrication industrielle.

Figure 21

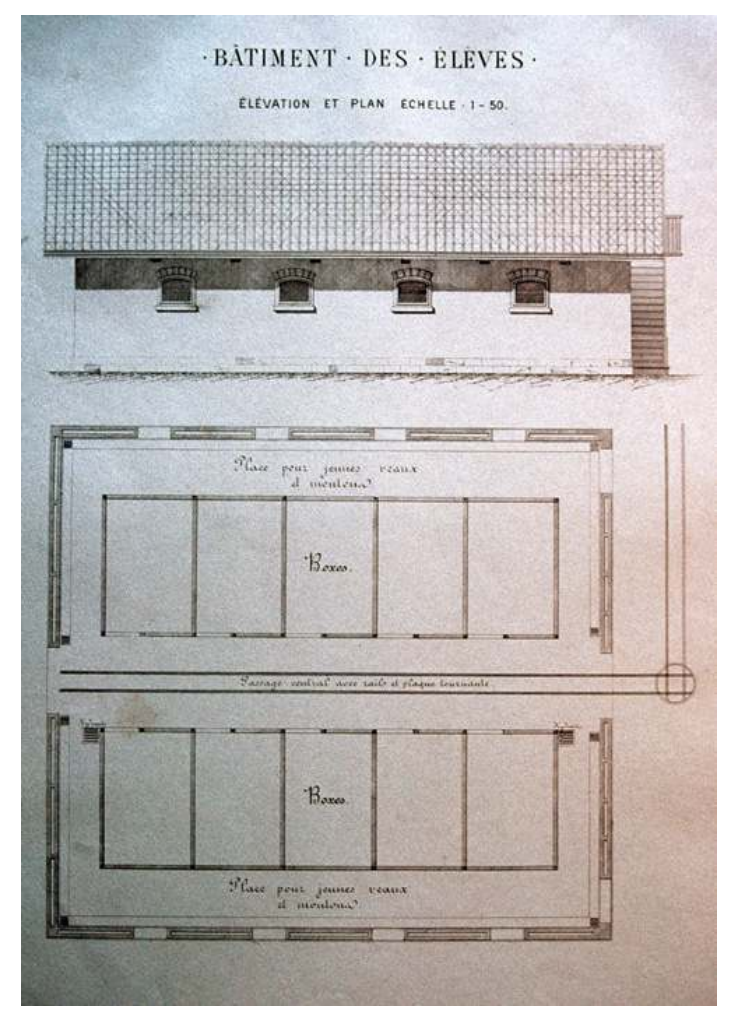

Album des plans des constructions agricoles élevées en 1878 sur le domaine de Beau-Cèdre, bâtiment pour le jeune bétail, élévation et plan.

Collection privée.

Les boxes étaient équipés d'une mangeoire à crémaillère dont on réglait la hauteur en fonction de l'âge et de la taille des bêtes. Ceux de la rangée orientale, peut-être réservés aux poulains, possédaient un sol abaissé formant un creux probablement destiné, comme 
le précise Louis Bouchard-Huzard, «à être rempli de marne, que l'on retire de temps en temps, lorsqu'elle est bien imprégnée d'urine ${ }^{15} »\left(\right.$ fig. $\left.{ }^{\circ} \mathbf{2 2}\right)$.

Figure 22

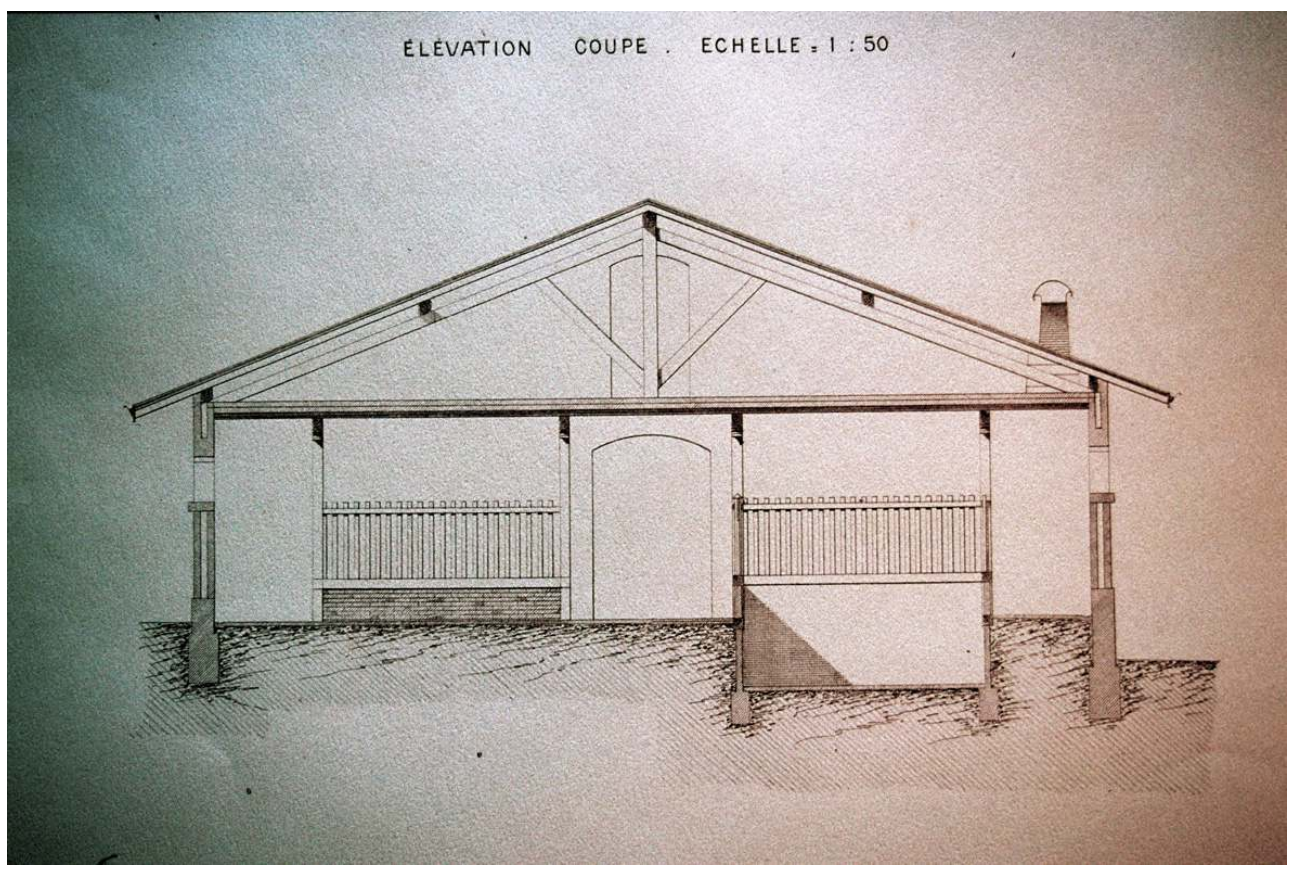

Album des plans des constructions agricoles élevées en 1878 sur le domaine de Beau-Cèdre, bâtiment pour le jeune bétail, coupe transversale, les boxes de la rangée orientale ayant un sol abaissé.

Collection privée.

\section{Le hangar}

Un vaste hangar aujourd'hui disparu fut édifié en 1878 au nord du bâtiment précédent. Construit selon un "système G. Auberjonois ", avec une structure à poteaux largement ouverte, il s'adossait au mur de clôture de la propriété (fig. $\mathbf{n}^{\circ} \mathbf{2 3}$ ). Il contenait un pont de déchargement transversal, accessible depuis la rue du village grâce à la pente, ainsi que divers espaces réservés au stockage et à la manutention (bottelage...). 


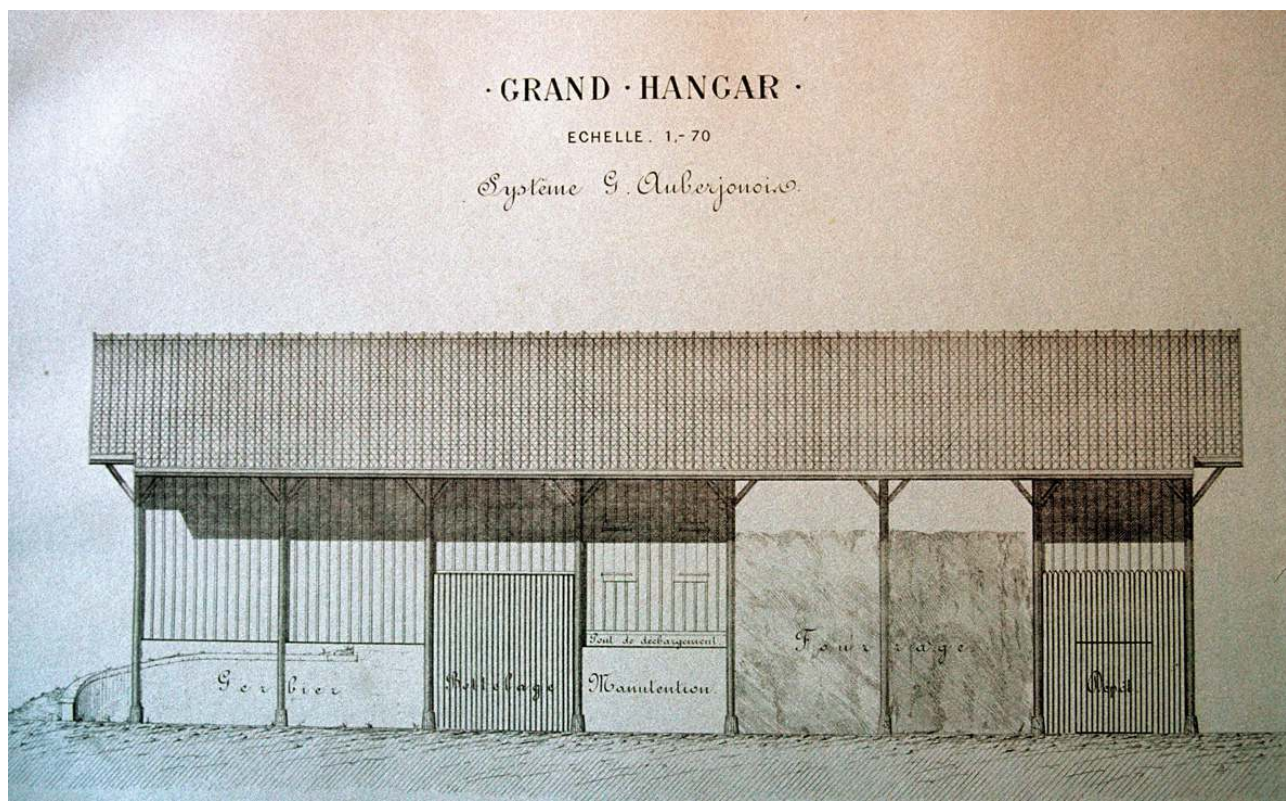

Album des plans des constructions agricoles élevées en 1878 sur le domaine de Beau-Cèdre, hangar construit selon un «système G. Auberjonois ».

Collection privée. la porcherie fut réhabilitée et transformée en bureau selon les plans de l'architecte et copropriétaire Jacques Gross, de même que le bâtiment des élèves qui devint une habitation. La vacherie, fort dégradée en 2012, fut malheureusement détruite en 2013. 


\section{Influence de Beau-Cèdre sur les constructions rurales du canton de Vaud}

Avant même son achèvement, le domaine de Beau-Cèdre est considéré comme un modèle dans le canton de Vaud. En 1881, le Bulletin de la Société vaudoise d'agriculture estime que « $\mathrm{M}$. Auberjonois aura rendu un vrai service à notre agriculture romande » si l'on suit son exemple. «Chacun, il est vrai, ne pourra pas y consacrer les sommes que Beau-Cèdre a dû coûter, mais ses étables, ses boxes pour les veaux, ses loges à porcs, son fenil et ses murs doubles sont certainement des objets d'études fort intéressants pour tous ceux qui sont dans le cas d'élever des constructions rurales ${ }^{16}{ }^{\prime}$.

Dans la région, quelques bâtiments vont s'inspirer de l'œuvre de Gustave Auberjonois. Par exemple, en 1897, le projet de l'architecte Charles Borgeaud pour une école d'agriculture à Aigle propose un système de chemin de fer et une porcherie pratiquement identique à celle de Beau-Cèdre. De même, la vacherie du château de Duilier, bâtie en 1905 par le bureau Morsier et Weibel, est subdivisée en quatre compartiments desservis par une voie ferrée. Finalement, en 1882, un "Atlas des bâtiments agricoles", édité à Lausanne, présente une vacherie fortement influencée par celle de Gustave Auberjonois.

Quant aux maisons paysannes, elles vont progressivement adopter plusieurs éléments testés à Beau-Cèdre, dans la mesure où leur prix est devenu accessible grâce à une production de type industriel. L'influence, dans ce cas, demeure indirecte et suit un mouvement général sensible à l'échelle internationale. Ainsi, les huisseries de fenêtres oblongues à châssis basculant, les cheminées de ventilation, les canaux d'évacuation du lisier, les «cornadis », les tuiles mécaniques, les poutrelles en $\mathrm{H}$ et les colonnettes en fonte vont se répandre dans l'architecture rurale de Suisse romande à partir des années 1900, avec des chronologies très variables selon les régions.

\section{Conclusion}

Même si une rue de Jouxtens-Mézery porte le nom de Beau-Cèdre, peu de personnes ont conscience de l'importance que revêtait jadis ce domaine. De nos jours, Gustave Auberjonois est essentiellement connu pour être le père du peintre René, le grand-père de l'écrivain et journaliste Fernand (1910-2004), voire l'arrière grand-père d'un acteur américain prénommé également René ! Pour rendre à cet homme ce qui lui est dû, citons l'article nécrologique paru dans La Terre vaudoise le 3 mars 1917 :

"Gustave Auberjonois, propriétaire de Beau-Cèdre, à Jouxtens, vient de mourir à l'âge de 80 ans. Il s'est vivement intéressé à l'agriculture comme praticien, spécialement dans la question de l'amélioration du bétail bovin en appuyant fidèlement, dès le début, l'œuvre entreprise dans ce sens par M. Viquerat. La porcherie, la laiterie et les cultures de BeauCèdre étaient très bien tenus. La mort d'un de ses fils qui aurait fait un agronome de mérite lui avait porté un coup fatal. M. Auberjonois laisse le souvenir du grand propriétaire, exploitant lui-même sa ferme, tradition qui malheureusement tend trop à disparaître dans notre canton. Par son exemple et ses engagements, il a largement contribué au développement de l'agriculture vaudoise. » 


\section{NOTES}

1. - RUEDI, Pierre. Jouxtens-Mézery, petite commune vaudoise, notes historiques, souvenirs anecdotiques. Jouxtens : P. Ruedi, 1967, p. 15.

2. - Pour tous les renseignements concernant les taxations des divers bâtiments de Beau-Cèdre : Archives cantonales vaudoises (ACV), GF 131/ 4, fol. 51 à 54, 153, 154, 159, 161 et 167.

3. - Archives privées de l'architecte Jacques Gross, copropriétaire de Beau-Cèdre, que je remercie vivement de m'avoir prêté ce document pour le photographier. Cet album de plans a été publié par la Librairie agricole de la maison rustique à Paris, B. Benda libraireéditeur à Lausanne et Sampson Low, Marston, Searle, \& Rivington à Londres.

4. - ACV, GEB 131, procès-verbal de taxation des immeubles de 1837, folio 14, $\mathrm{n}^{\circ} 11$.

5. - ACV, K XII a 975. Bulletin de la Société vaudoise d'agriculture et de viticulture, décembre $1881, \mathrm{n}^{\circ}$ 48, p. 93.

6. - BOUCHARD-HUZARD, Louis. Traité des constructions rurales. Paris: Léonce Laget, 1982, réimpression en fac-similé de l'édition de 1868-1870, tome 1, p. 138.

7. - ACV, K XII a 975. Bulletin de la Société vaudoise d'agriculture et de viticulture, décembre $1881, \mathrm{n}^{\circ}$ 48, p. 91 et 92 . Ce texte est un rapport de jury rédigé à la suite d'un concours pour la rédaction d'un manuel consacré aux bâtiments d'exploitation rurale. L'un des concurrents s'est inspiré de la vacherie de Beau-Cèdre, comme le précise le jury: «Nous trouvons exactement ici, pour le logement des bêtes, la disposition adoptée à Beau-Cèdre par M. Auberjonois, dans la belle ferme qu'il a construite il y a quelques années ".

8. - ACV, K XII a 975. Bulletin de la Société vaudoise d'agriculture et de viticulture, décembre $1881, \mathrm{n}^{\circ}$ 48, p. 93.

9. - BOUCHARD-HUZARD, Louis. Traité des constructions rurales. Paris: Léonce Laget, 1982, réimpression en fac-similé de l'édition de 1868-1870, tome 1, p. 92 et 93.

10. - BOUCHARD-HUZARD, Louis. Traité des constructions rurales. Paris: Léonce Laget, 1982, réimpression en fac-similé de l'édition de 1868-1870, tome 1, p. 136 à 138 et fig. 174 à 178.

11. - Lausanne, Bibliothèque cantonale universitaire, Manuscrits, Fonds Auberjonois (Famille), IS 2214. Lettre de René Auberjonois, 27 mars 1889.

12. - Lausanne, Bibliothèque cantonale universitaire, Manuscrits, Fonds Auberjonois (Famille), IS 2214. Lettre de Maurice Auberjonois, 24 mai 1899.

13. - BOUCHARD-HUZARD, Louis. Traité des constructions rurales. Paris: Léonce Laget, 1982, réimpression en fac-similé de l'édition de 1868-1870, tome 1, p. 193 à 194 et fig. 325 à 327.

14. - ACV, K XII a 975. Bulletin de la Société vaudoise d'agriculture et de viticulture, octobre $1882, \mathrm{n}^{\circ} 51$, p. 161.

15. - BOUCHARD-HUZARD, Louis. Traité des constructions rurales. Paris: Léonce Laget, 1982, réimpression en fac-similé de l'édition de 1868-1870, tome 1, p. 115 et 116, fig. 126.

16. - ACV, K XII a 975. Bulletin de la Société vaudoise d'agriculture et de viticulture, $\mathrm{n}^{\circ} 48$, décembre 1881, p. 94. 


\section{RÉSUMÉS}

Les bâtiments qui constituent le domaine de Beau-Cèdre à Jouxtens-Mézery, dans l'actuelle banlieue lausannoise, ont été édifiés de 1872 à 1891 par l'agronome Gustave Auberjonois qui en publia les plans à Paris, Londres et Lausanne. S'inspirant de divers modèles issus des traités d'architecture, dont la ferme impériale de Vincennes, et de ses expériences personnelles lors de séjours en Allemagne, en France et en Angleterre, Gustave Auberjonois a créé un ensemble radicalement novateur pour la Suisse romande, tant par son plan que par l'usage de matériaux modernes. Dès sa construction, ce domaine connut une certaine renommée, soulignée par la presse et les publications de l'époque.

The buildings which make up the estate of Beau-Cèdre at Jouxtens-Mézery, in the present suburb of Lausanne, were constructed between 1872 and 1891 by the agronomist Gustave Auberjonois who published the plans for them in Paris, London and Lausanne. Inspired by a number of designs derived from architectural studies, one of which being the imperial farm at Vincennes, and from his personal experiences from stays in Germany, France and England, Gustave Auberjonois created an ensemble which was radically innovative for Swiss Romandy both in design and in the use of modern materials. Ever since its construction this estate has had a certain reputation, highlighted by the press and the publications of the era.

INDEX

Mots-clés : Suisse romande, ferme modèle, agronomie, datation

\section{AUTEUR}

\section{ISABELLE ROLAND}

Historienne de l'architecture. Étude des maisons rurales en Suisse isabelleroland@bluewin.ch 